\title{
NUMERICAL SOLUTION OF THE SCALAR DOUBLE-WELL PROBLEM ALLOWING MICROSTRUCTURE
}

\author{
CARSTEN CARSTENSEN AND PETR PLECHÁČ
}

\begin{abstract}
The direct numerical solution of a non-convex variational problem $(P)$ typically faces the difficulty of the finite element approximation of rapid oscillations. Although the oscillatory discrete minimisers are properly related to corresponding Young measures and describe real physical phenomena, they are costly and difficult to compute.

In this work, we treat the scalar double-well problem by numerical solution of the relaxed problem $(R P)$ leading to a (degenerate) convex minimisation problem. The problem $(R P)$ has a minimiser $u$ and a related stress field $\sigma=D W^{* *}(\nabla u)$ which is known to coincide with the stress field obtained by solving $(P)$ in a generalised sense involving Young measures. If $u_{h}$ is a finite element solution, $\sigma_{h}:=D W^{* *}\left(\nabla u_{h}\right)$ is the related discrete stress field. We prove a priori and a posteriori estimates for $\sigma-\sigma_{h}$ in $L^{4 / 3}(\Omega)$ and weaker weighted estimates for $\nabla u-\nabla u_{h}$. The a posteriori estimate indicates an adaptive scheme for automatic mesh refinements as illustrated in numerical experiments.
\end{abstract}

\section{INTRODUCTION}

When we observe certain alloys near a critical temperature under a microscope we may see some fine layering called "microstructure". A mathematical model of these phenomena is possible by minimisation of an energy function [BJ87, BJ92]. The Ericksen-James energy density serves as an example

$$
W(F)=k_{1}\left(C_{11}+C_{22}-2\right)^{2}+k_{2} C_{12}^{2}+k_{3}\left(\left(C_{11}-C_{22}\right)^{2} / 2-\epsilon^{2}\right)^{2}
$$

where $C=F^{T} F=\left(C_{i j}\right)$ is the Cauchy deformation tensor and $F=\nabla u$ is the deformation gradient $\left(k_{1}, k_{2}, k_{3}, \epsilon\right.$ are material constants). In this paper, we consider the scalar double-well problem where

$$
W: \mathbb{R}^{n} \rightarrow \mathbb{R}, \quad F \mapsto\left|F-F_{1}\right|^{2} \cdot\left|F-F_{2}\right|^{2}
$$

for $F_{1}, F_{2} \in \mathbb{R}^{n}, F_{1} \neq F_{2}$. The scalar problem with (1.2) can be deduced from (1.1) in an anti-plane shear model and is considered in [Chi91, CC92, CL91, NW92, NW95]. A characteristic feature in this field of applications is that the energy

Received by the editor May 8, 1995 and, in revised form, May 3, 1996.

1991 Mathematics Subject Classification. Primary 65N15, 65N30, 35J70, 73C60.

Key words and phrases. Non-convex minimisation, Young measures, microstructure.

The work of the first author was supported by the EC under HCM ERB CH BG CT 920007, the work of the second author was supported under EPSRC grant GR/JO3466. 
density $W \geq 0$ is non-convex and $W(F)=0$ if and only if $F$ equals one of the two wells $F_{1}$ or $F_{2}$.

Definition 1.1. Let $\Omega \subset \mathbb{R}^{n}$ be a bounded Lipschitz domain with the boundary $\Gamma$ and let $\|u\|_{p}$ (resp. $\|u\|_{1, p}$ ) denote the norm in $L^{p}(\Omega)$ (resp. $W^{1, p}(\Omega)$ ) and $\nabla u(x) \in \mathbb{R}^{n}$ is the gradient of $u$.

Given functions $u_{0} \in W^{1, p}(\Omega), f, g \in L^{2}(\Omega)$, let $\mathcal{A}$ be a weakly closed subset of

$$
u_{0}+\mathcal{A}_{0}, \quad \mathcal{A}_{0}:=\left\{v \in W^{1, p}(\Omega): v=0 \text { a.e. on } \Gamma_{0}\right\},
$$

where $\Gamma_{0}$ is a measurable subset of $\Gamma$ with a positive surface measure. Let $\alpha \geq 0$ and define a functional $I$ on $W^{1, p}(\Omega)$ by

$$
\begin{aligned}
I(v):= & \int_{\Omega} W(\nabla v(x)) d x+\alpha \int_{\Omega}|f(x)-v(x)|^{2} d x \\
& -\int_{\Omega} g(x) \cdot v(x) d x \quad\left(v \in W^{1, p}(\Omega)\right) .
\end{aligned}
$$

Then the minimisation problem $(P)$ consists of seeking a minimiser $u$ of $I$ in $\mathcal{A}$.

As is illustrated in Young's well-known example, problems like $(P)$ may have many minimisers or may have no classical solution at all:

Example 1.1. Let $n=1$ and $\Omega=(0,1), W(s):=\left(1-s^{2}\right)^{2}$ (so $F_{j}=(-1)^{j}$ ), and let $\alpha=0, g=0$ and $u_{0}=0$. Consider $\mathcal{A}=u_{0}+\mathcal{A}_{0}$ with $\Gamma_{0}=\Gamma=\{0,1\}$. Then $0=\inf \{I(u): u \in \mathcal{A}\}$ is attained for all $u \in \mathcal{A}$ with $u= \pm 1$ almost everywhere in $(0,1)$. Thus, there are infinitely many solutions.

If $\alpha=1, g=0$ and $f \in L^{2}(\Omega)$ with $-1<f^{\prime}<1$ almost everywhere in $(0,1)$, then $0=\inf \{I(u): u \in \mathcal{A}\}$ but $I(u)>0$ for each $u \in \mathcal{A}$, so $(P)$ has no solution. See [NW95] for more details in the case $n=1$.

Although there is no classical solution one can generalise $(P)$ (as mentioned, e.g., in [KP91] and exploited in [Rou]) so that there is a generalised solution involving a displacement $u \in \mathcal{A}$ and a Young measure $\nu$ (cf., $\S 2$ below for details). Then there is a stress field

$$
\sigma:=\int_{\mathbb{R}^{n}} D W(A) d \nu(A)
$$

( $D W$ represents the gradient of $W$ ) which describes the stresses of minimising sequences in Problem $(P)$. Since the stress field is of practical interest, we emphasise the numerical approximation of (1.5) in this paper.

In scalar approximation problems, like Problem $(P)$, it is enough to consider a relaxation via a convex envelope [Dac89].

Definition 1.2. Let $W^{* *}$ be the convex envelope of $W$ and define the relaxed functional $I^{* *}$ on $W^{1, p}(\Omega)$ by

$$
\begin{aligned}
I^{* *}(v):= & \int_{\Omega} W^{* *}(\nabla v(x)) d x+\alpha \int_{\Omega}|f(x)-v(x)|^{2} d x \\
& -\int_{\Omega} g(x) \cdot v(x) d x \quad\left(v \in W^{1, p}(\Omega)\right) .
\end{aligned}
$$

Then Problem $(R P)$ consists of finding a minimiser $u$ of $I^{* *}$ in $\mathcal{A}$. 
Problem $(R P)$ has a minimiser $u$ which then defines the stress field

$$
\sigma=D W^{* *}(\nabla u)
$$

such that $\sigma$ in (1.7) actually equals $\sigma$ in (1.5) and is independent of $u$ amongst the solutions of $(R P)$; see [Fri94]. Therefore, to compute the stress field associated with Problem $(P)$ it is sufficient to solve numerically the degenerate convex problem $(R P)$ using a standard Galerkin scheme:

Definition 1.3. Let $\mathcal{A}_{0 h}$ be a discrete (i.e. finite dimensional) subspace of $\mathcal{A}_{0}$ and let $\mathcal{A}_{h}:=\left(u_{0}+\mathcal{A}_{0 h}\right) \cap \mathcal{A}$. Then Problem $\left(R P_{h}\right)$ consists of finding a minimiser $u_{h}$ of $I^{* *}$ in $\mathcal{A}_{h}$.

For the double-well problem (1.2), the discrete stress field

$$
\sigma_{h}:=D W^{* *}\left(\nabla u_{h}\right)
$$

is unique, i.e., $\sigma_{h}$ is independent of $u_{h}$ chosen as one of the solutions of Problem $\left(R P_{h}\right)$ (see Theorem 2 below).

From [Fri94] we can conclude convergence in measure of $\sigma_{h}$ to $\sigma$; see $\S 2$ below where we briefly recall some known facts on relaxation. In addition, for the doublewell problem at hand, we will prove strong convergence of the stress field in $\S 3$ (a slightly more general case) and in $\S 4$ (for $W$ as given in (1.2)). From Corollary 1 in $\S 4$, we obtain

$$
\left\|\sigma-\sigma_{h}\right\|_{4 / 3} \leq c \cdot \inf _{v_{h} \in \mathcal{A}_{h}}\left\|u-v_{h}\right\|_{1,4}
$$

for all solutions $u$ of $(R P)$ and $\sigma$ uniquely given in (1.7).

The situation is more involved for the displacement fields which will be studied in $\S 5$. Under some circumstances, like $\mathcal{A}=W_{0}^{1,4}(\Omega)$, there is uniqueness of solutions to $(R P)$ (even if $\alpha=0$ ) and weaker weighted error estimates for the displacement fields $u_{h}$ are established. In $\S 6$ we will prove convergence of Young measures $\nu_{h}$ which are constructed from the solution $u_{h}$ of the relaxed problem to describe oscillations arising in minimising sequences of Problem $(P)$. In some sense, the supports of $\nu_{h}$ converge strongly to $\operatorname{supp} \nu$, but there is only weak convergence for the coefficients, the volume fractions.

So far only a priori error estimates like (1.9) are considered in $\S 3-\S 6$. However, since higher regularity of $u$ does not seem to be known, a priori error estimates are of limited use in a practical error control: What is the meaning of the right-hand side in (1.9) if we have no information about the smoothness of $u$ at all? Therefore, a posteriori error estimates of the following type are important

$$
\left\|\sigma-\sigma_{h}\right\|_{4 / 3}+\alpha\left\|u-u_{h}\right\|_{2} \leq c \cdot\left(\sum_{T \in \mathcal{T}_{h}} \eta_{h}(T)\right)^{3 / 8}
$$

and will be established in $\S 7$. Quantities $\eta_{h}(T)$ are computable for each triangle $T$ (see $\S 7$ and $\S 8$ for details). From (1.10) we obtain some error control, even in cases where we have no a priori information, as illustrated in numerical experiments reported in $\S 8$. As $\eta_{h}(T)$ indicates an error distribution we will present an adaptive algorithm for automatic mesh refinement and apply it to numerical examples.

In summary, there is no loss of information if we consider the relaxed problem $(R P)$ instead of minimising $(P)$ (provided we are only interested in the macroscopic variables $u, \sigma$, and $\nu)$. The situation is, however, intrinsically more complicated in the vectorial case (i.e. for displacements $u: \mathbb{R}^{n} \rightarrow \mathbb{R}^{m}$ if $m, n \geq 2$ ). While the 
relaxation is again possible using the quasi-convex envelope $W^{\sharp}$ of $W$ (as in 1.1), $W^{\sharp}$ is explicitly known only in a few cases. Hence, one may face a direct numerical minimisation of Problem $(P)$ again.

\section{RELAXED AND Generalised PROBlem}

Although the minimisation problem $(P)$ may have no solution, there exist minimising sequences $\left(u_{j}\right)$ in $\mathcal{A}$, such that

$$
\lim _{j \rightarrow \infty} I\left(u_{j}\right)=\inf _{v \in \mathcal{A}} I(v) .
$$

Using standard arguments we observe that, (see, e.g., [Dac89] for details), first, $\left(u_{j}\right)$ is bounded in $W^{1,4}(\Omega)$ (in case of growth conditions like (3.1) below) and, second, $\left(u_{j}\right)$ has a weak-convergent subsequence (since $W^{1,4}(\Omega)$ is reflexive). The third property, the weak-lower semi-continuity of $I$, is lacking because $W$ is not convex [Dac89]. However, we may assume that $\left(u_{j}\right)$ is weakly convergent towards some $u$ in $W^{1,4}(\Omega)$,

$$
\left(u_{j}\right) \rightarrow u \text { (weakly) in } W^{1,4}(\Omega) .
$$

As a standard result in relaxation theory, see, e.g., [Dac89], the weak limit $u$ solves $(R P)$. To describe oscillations of $\left(u_{j}\right)$, we may consider a weak-convergence result for Radon measures.

Definition 2.1. Let $C_{0}\left(\mathbb{R}^{n}\right)$ be the Banach space of all continuous functions $f$ : $\mathbb{R}^{n} \rightarrow \mathbb{R}$ with $\lim _{|u| \rightarrow \infty} f(u)=0$. Let $\mathcal{M}(K)$ be the Banach space of Radon measures supported in $K \subseteq \mathbb{R}^{n}$ endowed with the norm $\|\mu\|_{\mathcal{M}}:=\|\mu\|_{\mathcal{M}(K)}=$ $\int_{K} d|\mu| . \quad L_{w}^{\infty}\left(\Omega ; \mathcal{M}\left(\mathbb{R}^{n}\right)\right)$ denotes the Banach space of families of measures $\left(\nu_{x}\right.$ : $x \in \Omega)$ with $\nu_{x} \in \mathcal{M}\left(\mathbb{R}^{n}\right)$ for almost all $x \in \Omega$ and such that

$$
\langle\nu, g\rangle: \Omega \rightarrow \mathbb{R}, \quad x \mapsto\left\langle\nu_{x}, g\right\rangle:=\int_{\mathbb{R}^{n}} g(A) d \nu_{x}(A)
$$

is measurable for each $g \in C_{0}\left(\mathbb{R}^{n}\right)$. The norm $L_{w}^{\infty}\left(\Omega ; \mathcal{M}\left(\mathbb{R}^{n}\right)\right)$ is $\operatorname{ess} \sup _{x \in \Omega}\left\|\nu_{x}\right\|_{\mathcal{M}}$. Let $\operatorname{YM}\left(\Omega ; \mathbb{R}^{n}\right)$ be the set of all $\nu \in L_{w}^{\infty}\left(\Omega ; \mathcal{M}\left(\mathbb{R}^{n}\right)\right)$ which are probability measures (i.e. $\nu_{x} \geq 0$ and $1=\left\|\nu_{x}\right\|_{\mathcal{M}}$ for almost all $x \in \Omega$ ). Then a sequence $\left(F_{k}\right)$ in $L^{p}\left(\Omega ; \mathbb{R}^{n}\right)$ is said to generate a Young measure $\nu$ if $\nu \in \mathrm{YM}\left(\Omega ; \mathbb{R}^{n}\right)$ and

$$
\left(g\left(F_{k}\right)\right) \rightarrow^{*}\langle\nu, g\rangle \quad \text { (weak star) in } L^{\infty}(\Omega) \quad \text { for all } g \in C_{0}\left(\mathbb{R}^{n}\right) .
$$

Theorem 1 ([Bal89]). Assume that the sequence $\left(u_{j}\right)$ is bounded in $W^{1, p}(\Omega)^{m}$, $1 \leq p \leq \infty$. Then there exists a subsequence $\left(u_{k}\right)$ such that $\left(\nabla u_{k}\right)$ generates a Young measure $\nu$. Moreover, if $g \in C\left(\mathbb{R}^{n}\right)$ and $g\left(\nabla u_{k}\right)$ is sequentially weakly relatively compact in $L^{1}(A), A$ a measurable subset of $\Omega$, then

$$
\left(g\left(\nabla u_{k}\right)\right) \rightarrow \int g(F) d \nu(F) \quad(\text { weakly }) \text { in } L^{1}(A) .
$$

The Young measure related to $(P)$ conveys information about oscillations of minimising sequences, cf., e.g., [Bal89, BJ92, Chi91, CC92, Fri94, NW95] and compare also $\S 6$ where we determine it from $u$ as approximate Young measures from the solutions $u_{h}$ of $\left(R P_{h}\right)$. The weak limit $u$ in (2.1) is linked to the Young measure $\nu$ by

$$
\nabla u(x)=\left\langle\nu_{x}, \mathrm{Id}\right\rangle .
$$


Remark 2.1. The relaxed problem $(R P)$ is connected with a generalised Problem $(G P)$ (cf., e.g., [KP91, Rou, NW92]) which (in the scalar case) consists of finding a minimiser $\left(u, \bar{\nu}_{x}\right) \in \mathcal{A} \times \operatorname{YM}\left(\Omega ; \mathbb{R}^{n}\right)$ of

$$
\mathcal{I}\left(v, \nu_{x}\right):=\int_{\Omega}\left\langle W, \nu_{x}\right\rangle d x+\alpha \int_{\Omega}|f(x)-v(x)|^{2} d x-\int_{\Omega} g(x) \cdot v(x) d x
$$

amongst all $\left\{v \in W^{1, p}(\Omega), \nu_{x} \in \mathrm{YM}\left(\Omega ; \mathbb{R}^{n}\right) ; \nabla v(x)=\left\langle\nu_{x}, \mathrm{Id}\right\rangle\right\}$. Then one can verify $W^{* *}(\nabla u)=\left\langle W, \nu_{x}\right\rangle$ for almost all $x \in \Omega$ and obtain the mentioned connections between $(P),(R P)$ and $(G P)$.

Remark 2.2. We refer to [DK91, KP91, Ped92] for the Young measure and its generating sequence in the vectorial case. We emphasise that (2.2) is not sufficient to ensure that $\nu$ is generated by a sequence of gradients with a weak limit $u$.

As part of a generalised solution of $(P)$, the Young measure $\nu$ allows a computation of a weak limit of stresses corresponding to minimising sequences: Define $\sigma$ by $(1.5)$ and $\sigma_{j}:=D W\left(\nabla u_{j}\right)$ where $u_{j}$ is a minimising sequence of $I$. Then we have convergence $\sigma_{j} \rightarrow \sigma$ in measure as proved in [Fri94] in a scalar case (under affine boundary conditions and $\alpha=0, g=0)$. Moreover, the stress field (1.5) equals $D W^{* *}(\nabla u)$ whenever $u$ solves $(R P)$. This relation emphasises the physical meaning of $\sigma$ in (1.5): $\sigma$ is the macroscopic stress field and can be measured in physical processes.

We refer to $\S 5$ for details on the gradients and to $\S 6$ for details on the Young measures.

\section{Numerical treatment of $(R P)$}

We will show in $\S 4$ that $W$ (as given in (1.2)) satisfies the following hypothesis assumed throughout this section.

Definition 3.1. Assume that $W: \mathbb{R}^{n} \rightarrow \mathbb{R}$ is $C^{1}$ and that there exist positive constants $c_{1}, \ldots, c_{5}$ and $p, q, r, s \in(1, \infty)$ with $\max \{1+q, 2 n /(n+2)\} \leq p$ such that, for all $E, F \in \mathbb{R}^{n}$,

$$
\begin{gathered}
\max \left\{c_{1}|F|^{p}-c_{2}, 0\right\} \leq W(F) \leq c_{3}+c_{4}|F|^{p}, \\
\left|D W^{* *}(F)\right| \leq c_{5} \cdot\left(1+|F|^{q}\right), \\
\left|D W^{* *}(F)-D W^{* *}(E)\right|^{r} \leq c_{6} \cdot\left(1+|F|^{s}+|E|^{s}\right) \\
\cdot\left(D W^{* *}(F)-D W^{* *}(E)\right)(F-E) .
\end{gathered}
$$

Let $\Sigma: \mathbb{R}^{n} \rightarrow \mathbb{R}^{n}, F \mapsto D W^{* *}(F)^{T}$.

Theorem 2. Under the hypothesis of Definition 3.1 there exist minimisers $u$ (resp. $u_{h}$ ) of (1.6) in $\mathcal{A}$ (resp. $\left.\mathcal{A}_{h}\right)$. The stresses and discrete stresses are uniquely determined, i.e., if $u$ and $v$ are two solutions of $(R P)\left(\right.$ resp. $\left.\left(R P_{h}\right)\right)$, then

$$
\Sigma(\nabla u)=\Sigma(\nabla v) \text { a.e. in } \Omega \text {. }
$$

Furthermore, if $1+s / p \leq t<r$, then

$$
\left\|\sigma-\sigma_{h}\right\|_{r / t}^{r}+\alpha\left\|u-u_{h}\right\|_{2}^{2} \leq c_{7} \cdot \inf _{v_{h} \in \mathcal{A}_{h}}\left(\alpha\left\|u-v_{h}\right\|_{2}^{2}+\left\|\nabla u-\nabla v_{h}\right\|_{r /(r-t)}^{r /(r-1)}\right)
$$


for $\sigma:=\Sigma(\nabla u)$ and $\sigma_{h}:=\Sigma\left(\nabla u_{h}\right)$ provided $u \in W^{1, r /(r-t)}(\Omega)$ solves $(R P)$ and $u_{h}$ $\in W^{1, r /(r-t)}(\Omega)$ solves $\left(R P_{h}\right)$. The constant $c_{7}>0$ depends on $\Omega, \Gamma, \Gamma_{0}, c_{1}, \ldots, c_{6}$ and $p, q, r, s$ only.

Proof. Since $W^{* *}$ inherits the growth conditions (3.1), the convex minimisation problem $(R P)$ has a minimiser. Given two solutions $u$ and $v$ of $(R P)$ the Gateauxderivative of $I^{* *}$ in the direction $u-v$ is zero either at $u$ or $v$. The difference between these two identities shows

$$
\int_{\Omega}(\Sigma(\nabla u)-\Sigma(\nabla v))^{T} \cdot \nabla(u-v) d x \leq-\alpha \int_{\Omega}|u-v|^{2} d x \leq 0 .
$$

Note that the integrand $(\Sigma(\nabla u)-\Sigma(\nabla v))^{T} \cdot \nabla(u-v)$ is non-negative by (3.3) and belongs to $L^{1}(\Omega)$ by (3.1), (3.2) and $q+1 \leq p$. Therefore, we infer that the integral is zero and, moreover, the integrand is pointwise zero almost everywhere in $\Omega$. Applying (3.3) to $F=\nabla u(x)$ and $E=\nabla v(x)$ we obtain $\Sigma(\nabla u(x))=\Sigma(\nabla v(x))$ for almost all $x \in \Omega$. The same arguments prove uniqueness of $\sigma_{h}$ as well. Hence, we may set $\sigma:=\Sigma(\nabla u)$ and $\sigma_{h}:=\Sigma\left(\nabla u_{h}\right)$ for some solution $u$ and $u_{h}$ of $(R P)$ and $\left(R P_{h}\right)$, respectively.

We now prove some standard a priori bounds for $u$ and $u_{h}$. The convex envelope $W^{* *}$ satisfies the same growth conditions as $W$ so we find from $u_{0} \in \mathcal{A}_{h}$ that

$$
c_{1}\left\|\nabla u_{h}\right\|_{p}^{p}-c_{2} \operatorname{meas}(\Omega)-\|g\|_{2}\left\|u_{h}\right\|_{2} \leq I^{* *}\left(u_{h}\right) \leq I^{* *}\left(u_{0}\right) .
$$

Since $1 / 2 \geq 1 / p-1 / n$ the embedding $W^{1, p}(\Omega) \hookrightarrow L^{2}(\Omega)$ is bounded by some $C_{1}>$ 0 . As $\Gamma_{0}$ has positive surface measure, Poincaré's inequality shows $C_{2}\left\|u_{h}\right\|_{1, p} \leq$ $\left\|\nabla u_{h}\right\|_{p}$. Hence,

$$
c_{1} C_{2}^{p}\left\|u_{h}\right\|_{1, p}^{p}-C_{1}\|f\|_{2}\left\|u_{h}\right\|_{1, p} \leq I^{* *}\left(u_{0}\right)+c_{2} \operatorname{meas}(\Omega),
$$

and so $\left\|u_{h}\right\|_{1, p} \leq c_{8}$ where $c_{8}$ is the positive root of

$$
c_{1} C_{2}^{p} \cdot c_{8}^{p}-C_{1}\|f\|_{2} \cdot c_{8}-I^{* *}\left(u_{0}\right)-c_{2} \operatorname{meas}(\Omega)=0 .
$$

The same arguments show that $\|u\|_{1, p} \leq c_{8}$ as well.

In the next step we derive bounds for the stresses. Taking the power $1 / t$ in (3.3) we obtain

$$
\left\|\sigma-\sigma_{h}\right\|_{r / t}^{r / t} \leq c_{6}^{1 / t} \int_{\Omega}\left(1+|\nabla u|^{s}+\left|\nabla u_{h}\right|^{s}\right)^{1 / t}\left(\left(\sigma-\sigma_{h}\right)\left(\nabla u-\nabla u_{h}\right)\right)^{1 / t} d x .
$$

By Hölder's inequality with exponents $t$ and $t^{\prime}, \frac{1}{t}+\frac{1}{t^{\prime}}=1$, on the right-hand side and by taking the power $t$,

$$
\left\|\sigma-\sigma_{h}\right\|_{r / t}^{r} \leq c_{6}\left\|1+|\nabla u|^{s}+\left|\nabla u_{h}\right|^{s}\right\|_{t^{\prime}}^{t} \cdot\left\|\left(\sigma-\sigma_{h}\right)^{T} \cdot\left(\nabla u-\nabla u_{h}\right)\right\|_{1} .
$$

Since $\nabla u$ and $\nabla u_{h}$ are bounded in $L^{p}(\Omega)^{n}$ by $c_{8}$, we obtain with $s t^{\prime} / t \leq p$

$$
\begin{aligned}
\left\|1+|\nabla u|^{s}+\left|\nabla u_{h}\right|^{s}\right\|_{t^{\prime}}^{t} & \leq\left(\int_{\Omega} 3^{t^{\prime} / t}\left(1+|\nabla u|^{p}+\left|\nabla u_{h}\right|^{p}\right) d x\right)^{t / t^{\prime}} \\
& \leq 3\left(\operatorname{meas}(\Omega)+2 c_{8}^{p}\right)^{t / t^{\prime}}=: c_{9} / c_{6} .
\end{aligned}
$$

Using this inequality and $0 \leq\left(\sigma-\sigma_{h}\right)^{T} \cdot\left(\nabla u-\nabla u_{h}\right)$ in (3.6), we obtain

$$
\left\|\sigma-\sigma_{h}\right\|_{r / t}^{r} \leq c_{9} \int_{\Omega}\left(\sigma-\sigma_{h}\right)^{T} \cdot\left(\nabla u-\nabla u_{h}\right) d x .
$$


The Galerkin orthogonality,

$$
\int_{\Omega}\left(\sigma-\sigma_{h}\right)^{T} \cdot \nabla v_{h} d x+2 \alpha \int_{\Omega}\left(u-u_{h}\right) v_{h} d x=0 \quad\left(v_{h} \in \mathcal{A}_{0 h}\right)
$$

leads, for each $v_{h} \in \mathcal{A}_{h}$, in (3.7) to

$$
\left\|\sigma-\sigma_{h}\right\|_{r / t}^{r} \leq c_{9} \int_{\Omega}\left(\sigma-\sigma_{h}\right)^{T} \cdot\left(\nabla u-\nabla v_{h}\right) d x+2 \alpha \int_{\Omega}\left(u-u_{h}\right)\left(u_{h}-v_{h}\right) d x .
$$

By Cauchy's and Hölder's inequalities

$$
\begin{gathered}
\left\|\sigma-\sigma_{h}\right\|_{r / t}^{r}+2 \alpha\left\|u-u_{h}\right\|_{2}^{2} \leq c_{9}\left\|\sigma-\sigma_{h}\right\|_{r / t}\left\|\nabla u-\nabla v_{h}\right\|_{r /(r-t)} \\
+2 \alpha\left\|u-u_{h}\right\|_{2}\left\|u-v_{h}\right\|_{2}
\end{gathered}
$$

and from Young's inequality $\left(x y \leq x^{r} / r+y^{r^{\prime}} / r^{\prime}\right.$ for $\left.x, y \geq 0,1 / r+1 / r^{\prime}=1\right)$ follows

$$
\left\|\sigma-\sigma_{h}\right\|_{r / t}^{r}+2 \alpha\left\|u-u_{h}\right\|_{2}^{2} \leq c_{9}^{r^{\prime}}(2 / r)^{1 /(r-1)}\left\|\nabla u-\nabla v_{h}\right\|_{r /(r-t)}^{r^{\prime}}+2 \alpha\left\|u-v_{h}\right\|_{2}^{2}
$$

which is (3.4).

Remark 3.1. Whenever $\alpha>0$, then $(R P)$ and $\left(R P_{h}\right)$ have unique solutions. If $\alpha=0$ this is in general false, see Example 1.1; however, see $\S 5$ for some uniqueness results.

Remark 3.2. In the discrete problem $\left(P_{h}\right)$, minimisers exist (according to compactness arguments in the finite dimensional case); but they are difficult to compute. This is mainly caused by a cluster of local minimisers around a global minimiser which have almost the same (minimal) energy. Moreover, given an approximation to a local minimiser, it is not easily verified whether this is a global minimiser or a nearby local minimiser. This situation cannot happen in the computation of solutions to $\left(R P_{h}\right)$ since, by convexity of $I^{* *}$, each local minimiser is a global minimiser.

Remark 3.3. If, in addition, $t \leq r(1-1 / p)$, then $u \in W^{1, p}(\Omega)$ is bounded in $W^{1, r /(r-t)}(\Omega)$ and the right-hand side of (3.4) tends to zero as $h \rightarrow 0$. Otherwise, convergence is not guaranteed a priori because, to the knowledge of the authors, higher regularity of $u$ has not yet been established for $n>1$; cf., [NW95] for $n=1$.

Remark 3.4. In this paper we compute the convex envelope of $W$ analytically for (1.2). In other cases, the convex envelope $W^{* *}$ can be approximated numerically (see, e.g., [BC94]).

Before we continue with the double-well problem in $\S 4$, let us consider a related (relaxed) problem, analysed in [Fre90], which has applications in elastoplastic antiplane shear [BP90, GT81] and optimal shape design [GKR86].

Example 3.1. Let $0<t_{1}<t_{2}$ and $0<\mu_{2}<\mu_{1}$ be positive real numbers that satisfy $t_{1} \mu_{1}=t_{2} \mu_{2}$. We define a $C^{1}$ function $\psi(t)$ with the following properties: $\psi(0)=0$, and for all $t \geq 0$

$$
\psi^{\prime}(t):= \begin{cases}\mu_{1} \cdot t & \text { if } 0 \leq t \leq t_{1}, \\ t_{1} \mu_{1}=t_{2} \mu_{2} & \text { if } t_{1} \leq t \leq t_{2}, \\ \mu_{2} \cdot t & \text { if } t_{2} \leq t .\end{cases}
$$

Under the assumption $\mu_{2}<\mu_{1}$ the function $\psi$ is convex and the function $W(F):=$ $\psi(|F|)$ defined for all $F \in \mathbb{R}^{n}$ satisfies the assumptions of Definition 3.1. Indeed, 
convexity of $\psi$ implies $W^{* *}=W$, and $D W(F)=\phi(|F|) \cdot F$ where $\phi(0):=0$ and $\phi(t):=\psi^{\prime}(t) / t$ for $t>0$. It is essential to note that $\mu_{2} \leq \phi \leq \mu_{1}$ and $\phi$ is a monotone decreasing function. Simple calculations yield constants in the assumptions of Definition 3.1: $c_{1}=\mu_{2} / 2, c_{2}=c_{3}=0, c_{4}=\mu_{1} / 2, c_{5}=\mu_{1}$, $c_{6}=1 / \mu_{1}$ and parameters $p=r=2$. The assumptions are satisfied for all $q \geq 1$ and $s \geq 0$. While the proof of (3.1) and (3.2) is straightforward, we sketch the proof of (3.3): For $E, F \in \mathbb{R}^{n}$ set $\sigma:=D W^{* *}(E)=\alpha E$ and $\tau:=D W^{* *}(E)=\beta F$, where $\alpha=\phi(|E|)$ and $\beta=\phi(|F|)$. Assuming $0<|E| \leq|F|$ we infer

$$
\begin{array}{r}
\left(D W^{* *}(F)-D W^{* *}(E)\right)(F-E)=(\sigma-\tau) \cdot(\sigma / \alpha-\tau / \beta) \\
\quad=\frac{1}{2}\left(\alpha^{-1}+\beta^{-1}\right)|\sigma-\tau|^{2}+\frac{1}{2}\left(\alpha^{-1}-\beta^{-1}\right)\left(|\sigma|^{2}-|\tau|^{2}\right) .
\end{array}
$$

According to $|E| \leq|F|$ we have $|\sigma| \leq|\tau|$ and $\mu_{2} \leq \beta \leq \alpha \leq \mu_{1}$, therefore the last term on the right-hand side is non-negative. This proves (3.3) with $c_{6}=1 / \mu_{1}$ and $s=0$.

The proof of Theorem 2 holds true for $t=1$ and $s=0$ as well (it is simpler) so that Theorem 2 shows uniqueness results for the stress as in [Fre90, Proposition 2.3 ] and gives an error estimate

$$
\left\|\sigma-\sigma_{h}\right\|_{2}^{2}+\alpha\left\|u-u_{h}\right\|_{2}^{2} \leq c_{7} \cdot \inf _{v_{h} \in \mathcal{A}_{h}}\left(\alpha\left\|u-v_{h}\right\|_{2}^{2}+\left\|\nabla u-\nabla v_{h}\right\|_{2}^{2}\right)
$$

for the stress variables in any dimension (cf., [Fre90, Theorem 4.1] for a similar result in the case $n=1$ ).

\section{Double-Well problem as a special CASE}

We start with a reformulation of $W$ as defined in (1.2) to give an explicit representation of $W^{* *}$ and to verify the hypothesis in Definition 3.1.

Definition 4.1. Let $A:=\left(F_{2}-F_{1}\right) / 2 \neq 0$ and $B:=\left(F_{1}+F_{2}\right) / 2$. Define $(s)_{+}:=$ $\max \{s, 0\}$ for any real $s$.

Proposition 1. If $W$ is defined in (1.2), then for all $F \in \mathbb{R}^{n}$,

$$
\begin{gathered}
W(F)=\left(|F-B|^{2}-|A|^{2}\right)^{2}+4\left(|A|^{2} \cdot|F-B|^{2}-\left[A^{T} \cdot(F-B)\right]^{2}\right), \\
W^{* *}(F)=\left[\left(|F-B|^{2}-|A|^{2}\right)_{+}\right]^{2}+4\left(|A|^{2} \cdot|F-B|^{2}-\left[A^{T} \cdot(F-B)\right]^{2}\right) .
\end{gathered}
$$

Proof. The identity (4.1) is proved by direct calculations; we omit the details. To prove (4.2), let $\omega(F)=\omega_{1}(F)+\omega_{2}(F)$ denote the right-hand side of (4.2).

Note that $\omega_{1}(F):=\left[\left(|F-B|^{2}-|A|^{2}\right)_{+}\right]^{2}$ is convex in $F$ because $\omega_{1}$ is the composition of a convex and monotone function $s \mapsto\left(s^{2}-|A|^{2}\right)_{+}^{2}$ and the convex function $F \mapsto|F-B|$. The function $\omega_{2}$ is smooth, its second Fréchet derivative is

$$
D^{2} \omega_{2}(F)(G, H)=8\left(|A|^{2} G^{T} \cdot H-\left(A^{T} \cdot G\right)\left(A^{T} \cdot H\right)\right)
$$

and, from the Cauchy-Schwarz inequality, $D^{2} \omega$ is a positive semi-definite bilinear form. Hence, $\omega_{2}$ is convex and so is $\omega$. 
Since $\omega \leq W$ and $\omega$ is convex, we have $\omega \leq W^{* *} \leq W$. To prove $\omega \geq W^{* *}$ we consider $F_{0} \in \mathbb{R}^{n}$ with $\omega\left(F_{0}\right)<W\left(F_{0}\right)$, whence $\left|F_{0}-B\right|<|A|$ and

$$
\omega\left(F_{0}\right)=4\left(|A|^{2}\left|F_{0}-B\right|^{2}-\left[A^{T} \cdot\left(F_{0}-B\right)\right]^{2}\right) .
$$

Since $\left|F_{0}-B\right|<|A|$, we conclude that there exist real $\alpha_{1}<0<\alpha_{2}$ with

$$
\left|F_{j}-B\right|=|A| \quad \text { and } \quad F_{j}:=F_{0}+\alpha_{j} \cdot A \quad(j=1,2) .
$$

Hence, for $j=1,2$, we can calculate

$$
W\left(F_{j}\right)=4\left(|A|^{2}\left|F_{0}-B+\alpha_{j} A\right|^{2}-\left[A^{T} \cdot\left(F_{0}-B+\alpha_{j} A\right)\right]^{2}\right)=\omega\left(F_{0}\right) .
$$

Let $\lambda=\alpha_{2} /\left(\alpha_{2}-\alpha_{1}\right)$. Then

$$
F_{0}=\lambda F_{1}+(1-\lambda) F_{2} \quad \text { and } \quad \omega\left(F_{0}\right)=\lambda W\left(F_{1}\right)+(1-\lambda) W\left(F_{2}\right)
$$

and so, since $W^{* *}$ is convex and $W^{* *} \leq W$,

$$
W^{* *}\left(F_{0}\right) \leq \lambda W^{* *}\left(F_{1}\right)+(1-\lambda) W^{* *}\left(F_{2}\right) \leq \lambda W\left(F_{1}\right)+(1-\lambda) W\left(F_{2}\right)=\omega\left(F_{0}\right),
$$

which concludes the proof.

In the second step we prove (3.3).

Definition 4.2. Let $A:=\left(F_{2}-F_{1}\right) / 2 \neq 0, A_{0}:=|A|^{-1} \cdot A$, and $B:=\left(F_{1}+F_{2}\right) / 2$. Let $\mathbb{I}$ denote the $n \times n$-unit matrix and let $\otimes$ be the dyadic product of two vectors. Then

$$
\mathbb{P}=\mathbb{I}-A_{0} \otimes A_{0}
$$

denotes the orthogonal projection in $\mathbb{R}^{n}$ onto $\operatorname{span}\{A\}^{\perp}$, the orthogonal complement of $\operatorname{span}\{A\}$. Define the function $\Sigma: \mathbb{R}^{n} \rightarrow \mathbb{R}^{n}$ by

$$
\Sigma(F):=4\left[\left(|F-B|^{2}-|A|^{2}\right)_{+} \cdot \mathbb{I}+2|A|^{2} \mathbb{P}\right](F-B) \quad\left(F \in \mathbb{R}^{n}\right) .
$$

Proposition 2. For any $F, G \in \mathbb{R}^{n}$ and

$$
\xi:=\left(|F-B|^{2}-|A|^{2}\right)_{+}, \quad \eta:=\left(|G-B|^{2}-|A|^{2}\right)_{+},
$$

we have $\Sigma(F)=D W^{* *}(F)^{T}$ and

$$
|\Sigma(F)-\Sigma(G)|^{2} \leq 8\left(\eta+\xi+2|A|^{2}\right) \cdot(\Sigma(F)-\Sigma(G))^{T} \cdot(F-G) .
$$

Proof. By direct calculations, $\Sigma(F)=D W^{* *}(F)^{T}$ and we focus on (4.6). Throughout the proof let $U:=F-B, V:=G-B$ and let the real numbers $e_{1}, \ldots, e_{8}$ denote certain expressions as defined below. Since (4.6) is symmetric in $F$ and $G$, we assume (without loss of generality) that $0 \leq \eta \leq \xi$, i.e., $|V| \leq|U|$. It follows from the definition of $\Sigma(F)$ and $\Sigma(G)$ that

$$
\begin{aligned}
(\Sigma(F)-\Sigma(G))^{T} \cdot(F-G)= & 8|A|^{2} \cdot(F-G)^{T} \cdot \mathbb{P} \cdot(F-G) \\
& +4(U-V)^{T} \cdot(\xi U-\eta V) \\
= & : e_{1}+e_{2} .
\end{aligned}
$$

Writing $\xi U-\eta V=\frac{\xi+\eta}{2}(U-V)+\frac{\xi-\eta}{2}(U+V)$ in $e_{2}$ we obtain

$$
e_{2}=2(\xi+\eta)|U-V|^{2}+2(\xi-\eta)\left(|U|^{2}-|V|^{2}\right)=: e_{3}+e_{4} .
$$


Note that $e_{1} \geq 0, e_{3} \geq 0$ and $e_{4}:=2(\xi-\eta)\left(|U|^{2}-|V|^{2}\right) \geq 0$ (because of $0 \leq \eta \leq \xi$ ) and we have verified

$$
e_{1}+e_{3}+e_{4}=(\Sigma(F)-\Sigma(G))^{T} \cdot(F-G) .
$$

By definition of $\Sigma(F), \Sigma(G)$ again, we obtain

$$
\begin{aligned}
|\Sigma(F)-\Sigma(G)|^{2} & \leq\left.|8| A\right|^{2} \mathbb{P}(F-G)+4 \xi U-\left.4 \eta V\right|^{2} \\
& \leq 128|A|^{4}|\mathbb{P}(F-G)|^{2}+16|\xi U-\eta V|^{2}=: e_{5}+e_{6} .
\end{aligned}
$$

Since $\mathbb{P}$ is a symmetric projection,

$$
e_{5}=128|A|^{4}(F-G)^{T} \mathbb{P}(F-G)=16|A|^{2} e_{1} .
$$

Using the above splitting of $\xi U-\eta V$ again, we are led to

$$
e_{6}=16|\xi U-\eta V|^{2} \leq 8(\xi+\eta)^{2}|U-V|^{2}+8(\xi-\eta)^{2}|U+V|^{2}=: e_{7}+e_{8} .
$$

Note that

$$
e_{7}=8(\xi+\eta)^{2}|U-V|^{2}=4(\xi+\eta) e_{3} .
$$

We remark that $0 \leq \xi-\eta \leq|U|^{2}-|V|^{2}$ and $\frac{1}{2}|U+V|^{2} \leq|U|^{2}+|V|^{2} \leq \xi+\eta+2|A|^{2}$ (even if $\eta=0$ because then $|V| \leq|A|$ ). Hence,

$e_{8}=8(\xi-\eta)^{2}|U+V|^{2} \leq 16(\xi-\eta)\left(|U|^{2}-|V|^{2}\right)\left(\xi+\eta+2|A|^{2}\right)=8\left(\xi+\eta+2|A|^{2}\right) e_{4}$.

Gathering the above estimates for $e_{5}, e_{6}, e_{7}$ and $e_{8}$, we get from (4.8),

$$
|\Sigma(F)-\Sigma(G)|^{2} \leq e_{5}+e_{7}+e_{8} \leq 8\left(\xi+\eta+2|A|^{2}\right)\left(e_{1}+e_{3}+e_{4}\right) .
$$

According to (4.7), this proves (4.6).

The inequality (1.9) readily follows as a special case of Theorem 2 and we present it as a separate corollary.

Corollary 1. As defined in (1.2), W satisfies the hypothesis in Definition 3.1 with $p=4, q=3, r=2, s=2$. The conclusion of Theorem 2 is valid, in particular, for all $\rho$ with $1<\rho \leq 4 / 3$,

$$
\left\|\sigma-\sigma_{h}\right\|_{\rho}+\alpha\left\|u-u_{h}\right\|_{2} \leq \sqrt{2 c_{9}} \cdot \inf _{v_{h} \in \mathcal{A}_{h}}\left(\alpha\left\|u-v_{h}\right\|_{2}+\left\|\nabla u-\nabla v_{h}\right\|_{\rho /(\rho-1)}\right)
$$

for all $\sigma:=\Sigma(\nabla u)$ and $\sigma_{h}:=\Sigma\left(\nabla u_{h}\right)$ provided $u$ solves $(R P)$ and $u_{h}$ solves $\left(R P_{h}\right)$.

Proof. A rough estimation of $W$ (as given in (1.2)) shows that (3.1) holds for $p=4$, $c_{1}=1 / 8, c_{2}=c_{3}=8 \cdot \max \left\{\left|F_{1}\right|^{4},\left|F_{2}\right|^{4}\right\}$, and $c_{4}=8$. Moreover, (3.2) is true with $q=3$ and

$$
\left.c_{5}:=8 \max \left\{2, \frac{1}{3}|F|^{3}+|B|\left(|A|^{2}+|B|^{2}\right)+\frac{2}{3}\left(|A|^{2}+|B|\right)^{3 / 2}\right)\right\} .
$$

From Proposition 2, we obtain (3.3) with $r=2$ and $c_{6}:=16 \max \left\{1,|A|^{2}+2|B|^{2}\right\}$. Therefore, Theorem 2 applies to the double-well problem in question and concludes the proof. 


\section{REMARKS ON THE GRADIENTS IN THE DOUBLE-WELL PROBLEM}

In general, we can neither expect to have uniqueness of the displacement field nor (as a consequence) get bounds for the gradients, cf., Example 1.1. However, we obtain estimates in weighted norms and uniqueness of the gradients under some circumstances.

We start with an analogue of Proposition 2 and consider $W$ as defined in (1.2).

Proposition 3. For any $F, G \in \mathbb{R}^{n}$ and

$$
\xi:=\left(|F-B|^{2}-|A|^{2}\right)_{+}, \quad \eta:=\left(|G-B|^{2}-|A|^{2}\right)_{+},
$$

we have

$$
\begin{aligned}
8|A|^{2} & \cdot|\mathbb{P} F-\mathbb{P} G|^{2}+2(\xi+\eta)\left|A_{0}^{T} \cdot(F-G)\right|^{2}+2(\xi-\eta)^{2} \\
& \leq(\Sigma(F)-\Sigma(G))^{T} \cdot(F-G) .
\end{aligned}
$$

Proof. Arguing as in the proof of Proposition 2 we see that the three terms on the left-hand side in (5.1) are bounded by $e_{1}, e_{3}$ and $e_{4}$ while the right-hand side equals $e_{1}+e_{3}+e_{4}$.

Theorem 3. Two solutions $u$ and $v$ of $(R P)$ (resp. of $\left(R P_{h}\right)$ ) satisfy almost everywhere in $\Omega$

$$
\mathbb{P} \nabla u=\mathbb{P} \nabla v \quad \text { and } \quad\left(|\nabla u-B|^{2}-|A|^{2}\right)_{+}=\left(|\nabla v-B|^{2}-|A|^{2}\right)_{+},
$$

and for almost all $x \in \Omega$ with $\left(|\nabla u(x)-B|^{2}-|A|^{2}\right)_{+}=\left(|\nabla v(x)-B|^{2}-|A|^{2}\right)_{+}>0$, we have $\nabla v(x)=\nabla u(x)$.

The microstructure region for two solutions $u$ and $v$ are identical, i.e.,

$$
\{x \in \Omega:|\nabla u(x)-B| \leq|A|\}=\{x \in \Omega:|\nabla v(x)-B| \leq|A|\} .
$$

We have $u=v$ almost everywhere in

$$
\Omega_{0, A}:=\bigcup\left\{x \in \Omega:(x, y) \subset \Omega \text { and }(y-x)^{T} \cdot A=0 \text { for } y \in \Gamma_{0}\right\},
$$

where $(x, y):=\{\lambda x+(1-\lambda) y: 0<\lambda<1\}$ is the open interval between $x$ and $y$. In particular, $u=v$ in case $\Gamma=\Gamma_{0}$.

Proof. According to Theorem $2, \Sigma(\nabla u)=\Sigma(\nabla v)$ almost everywhere in $\Omega$, so that Proposition 3 shows the first part of the theorem. Define $w:=u-v$ and notice that $\nabla w \perp A$ almost everywhere in $\Omega$. Hence, there is some $a \in L^{1}(\Omega)$ with $\nabla w(x)=a(x) A$ for almost all $x \in \Omega$. Let $x$ denote a Lebesgue point of $\nabla w$ and let the ball $B(x, \epsilon)=\left\{y \in \mathbb{R}^{n}:|y-x|<\epsilon\right\}$ belong to $\Omega$. Consider $y \in B(x, \epsilon)$, $x \neq y$, with $(y-x)^{T} \cdot A=0$, i.e., $y \in x+\operatorname{span}\{A\}^{\perp}$. We know from the fine properties of Sobolev functions that $w$ is absolutely continuous on almost all (in the sense of the Lebesgue measure on $\mathbb{R}^{n-1}$ ) lines parallel to $x-y$ (cf., e.g., [EG92]). Since $\nabla w=a A, w$ is constant on almost every of these lines. We conclude that the function $a$ depends only on the $A$-direction of the argument, i.e., in each ball $B\left(x_{0}, \epsilon_{0}\right) \subset \Omega$, we have a function $b \in L^{1}(\mathbb{R})$ with $a(x)=b(x)\left(A^{T} \cdot\left(x-x_{0}\right)\right)$ for almost all $x \in B\left(x_{0}, \epsilon_{0}\right)$. Integration along straight lines in $B\left(x_{0}, \epsilon_{0}\right)$ now shows that $w$ is a function which depends (absolutely continuously) on the $A$-direction of the arguments only. Hence, even globally, $w$ is absolutely continuous and constant on components of the intersection of $\bar{\Omega}$ with hyperplanes in the direction $A$. 
If $x \in \Omega_{0, A}$ we have an interval $(x, y)$ that connects $x$ and $y$, such that $w$ is constant along $(x, y)$. The Dirichlet boundary condition gives $w(y)=0$, whence $w(x)=0$.

According to Theorem 3, the following coefficients are uniquely defined (i.e., they do not depend on the choice of the minimisers $u$ and $u_{h}$ ).

Definition 5.1. Let $u$ solve $(R P)$ and let $u_{h}$ solve $\left(R P_{h}\right)$; we denote $\xi:=$ $\left(|\nabla u-B|^{2}-|A|^{2}\right)_{+}$and $\xi_{h}:=\left(\left|\nabla u_{h}-B\right|^{2}-|A|^{2}\right)_{+}$. The microstructure region and its approximation are defined as the sets $\Omega_{m}:=\{x \in \Omega: \xi(x)=0\}$ and $\Omega_{m h}:=\left\{x \in \Omega: \xi_{h}(x)=0\right\}$, respectively.

Proposition 3 yields an error estimate for $\xi_{h}$ and $u_{h}$.

Theorem 4. There exists a positive constant $c_{10}$ which depends only upon I but not on $\mathcal{A}_{h}$, such that

$$
\begin{aligned}
\left\|\mathbb{P} \nabla u-\mathbb{P} \nabla u_{h}\right\|_{2}+\left\|\left|\xi+\xi_{h}\right|^{1 / 2} \cdot A_{0}^{T} \cdot\left(\nabla u-\nabla u_{h}\right)\right\|_{2}+\left\|\xi-\xi_{h}\right\|_{2}+\alpha\left\|u-u_{h}\right\|_{2} \\
\leq c_{10} \cdot \inf _{v_{h} \in \mathcal{A}_{h}}\left(\alpha\left\|u-v_{h}\right\|_{2}+\left\|\nabla u-\nabla v_{h}\right\|_{4}\right) .
\end{aligned}
$$

Proof. Let LHS denote the left-hand side in (5.2) multiplied by a generic constant. Apply Proposition 3 to $\nabla u(x)$ and $\nabla u_{h}(x)$ for almost all $x \in \Omega$ and integrate to verify

$$
L H S^{2} \leq \int_{\Omega}\left(\sigma-\sigma_{h}\right)^{T} \cdot\left(\nabla u-\nabla u_{h}\right) d x+\alpha\left\|u-u_{h}\right\|_{2}^{2} .
$$

According to (3.8), for any $v_{h} \in \mathcal{A}_{h}$, this gives

$$
\begin{aligned}
L H S^{2} & \leq \int_{\Omega}\left(\sigma-\sigma_{h}\right)^{T} \cdot\left(\nabla u-\nabla v_{h}\right) d x+2 \alpha \int_{\Omega}\left(u-u_{h}\right)\left(u_{h}-v_{h}\right) d x+\alpha\left\|u-u_{h}\right\|_{2}^{2} \\
& \leq\left\|\sigma-\sigma_{h}\right\|_{4 / 3}\left\|\nabla u-\nabla v_{h}\right\|_{4}+2 \alpha \int_{\Omega}\left(u-u_{h}\right)\left(u-v_{h}\right) d x-\alpha\left\|u-u_{h}\right\|_{2}^{2} .
\end{aligned}
$$

Then with Corollary 1, LHS $S^{2} \inf _{v_{h} \in \mathcal{A}_{h}}\left(\alpha\left\|u-v_{h}\right\|_{2}^{2}+\left\|\nabla u-\nabla v_{h}\right\|_{4}^{2}\right)$.

Remark 5.1. In the case $n=1$, Nicolaides and Walkington proved strong convergence of gradients for a slightly modified numerical method in [NW95]. The question of strong convergence of gradients in a Galerkin scheme under consideration here is still open even for $n=1$.

\section{Remarks on Young measures}

In the double-well problem (with $W$ given in (1.2)) the Young measure $\nu_{x}$ described in Theorem 1 is known as soon as we determine a solution of $(R P)$ :

Definition 6.1. For any $F \in \mathbb{R}^{n}$ with $|F-B|<|A|$ define $\lambda(F) \in[0,1]$ and $S_{ \pm}(F)$ by

$$
\begin{aligned}
\lambda(F) & :=\frac{1}{2}\left(1+A_{0}^{T} \cdot(F-B) \cdot\left(|A|^{2}-|\mathbb{P}(F-B)|^{2}\right)^{-1 / 2}\right), \\
S_{ \pm}(F) & :=B+\mathbb{P}(F-B) \pm\left(|A|^{2}-|\mathbb{P}(F-B)|^{2}\right)^{1 / 2} \cdot A_{0} .
\end{aligned}
$$




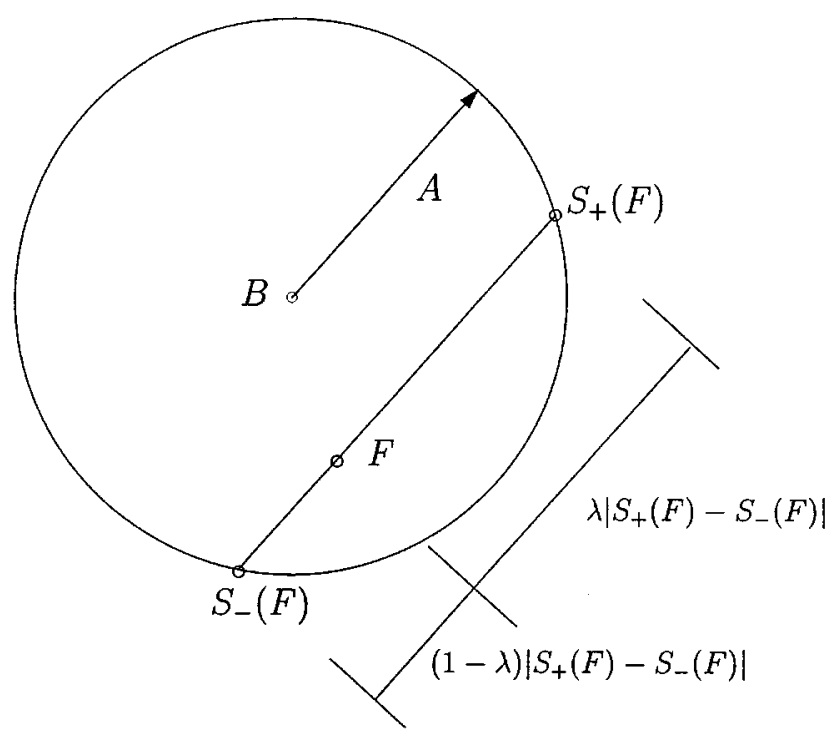

FIGURE 1. Construction of $\mu(F)$

For $F \in \mathbb{R}^{n}$, let $\delta_{F}$ be the Dirac measure with mass $F$ and let $\mu(F) \in \mathrm{YM}\left(\Omega ; \mathbb{R}^{n}\right)$ be

$$
\mu(F):= \begin{cases}\delta_{F} & \text { if }|A| \leq|F-B|, \\ \lambda(F) \cdot \delta_{S_{+}(F)}+(1-\lambda(F)) \cdot \delta_{S_{-}(F)} & \text { if }|F-B|<|A| .\end{cases}
$$

Remark 6.1. The geometric interpretation of the support $S_{ \pm}(F)$ of $\mu(F)$ is depicted in Fig. 1: If $|A| \leq|F-B|$, then $\mu(F)$ is a Dirac measure with the centre $F$. Otherwise $F$ lies in the ball around $B$ with radius $|A|$ and there are two points $S_{+}(F)$ and $S_{-}(F)$ on the surface which belong also to the straight line through $F$ in the direction $A$. The coefficient $\lambda(F)$ describes the location of $F$ on the interval $\left[S_{+}(F), S_{-}(F)\right]$.

Proposition 4. Assume that $\left(u_{j}\right)$ is a minimising sequence of $I$ in $\mathcal{A}$ which is weakly convergent to $u \in \mathcal{A}$ and which generates a Young measure $\nu$. Then u solves $(R P)$ and $\nu_{x}=\mu(\nabla u(x))$ for almost all $x \in \Omega$.

Proof. As in [Fri94] we conclude (with additional terms $f, g$ and under general boundary conditions which cause no essential difficulties) that any $\nu_{x}$ (as given by Theorem 1) satisfies (2.2) and

$$
W^{* *} \text { is affine on } \operatorname{conv} \operatorname{supp} \nu_{x} \text { and } \operatorname{supp} \nu_{x} \subseteq\left\{E \in \mathbb{R}^{n}: W(E)=W^{* *}(E)\right\}
$$

for almost all $x \in \Omega$. So it remains to fix such a point $x$ and prove that $F:=\nabla u(x)$ and (2.2) and (6.4) uniquely determine $\nu_{x}$ to be $\mu(F)$. It is obvious that $\nu_{x}=\delta_{F}$ in the case where the support of $\nu_{x}$ consists of one point only. So let us assume $C, D \in \operatorname{supp} \nu_{x}$ in the sequel with $C \neq D$. Then $W^{* *}$ is affine on $\operatorname{supp} \nu_{x}$. By using notation from the proof of Proposition 1, $W^{* *}=\omega_{1}+\omega_{2}$ and $\omega_{j}$ is affine on the interval $[C, D] \subset \mathbb{R}^{n}$. Since $\omega_{1}$ vanishes or is strictly convex, we conclude 
that $\omega_{1}(C)=\omega_{1}(D)=0$, whence $|C-B|,|D-B| \leq|A|$. Moreover, from (4.3) and $D^{2} \omega_{2}\left(\frac{1}{2}(D-C)\right)(D-C, D-C)=0$ we obtain equality in Cauchy-Schwarz's inequality $\left|A^{T} \cdot(D-C)\right|=|A| \cdot|D-C|$ and infer that $D-C$ is parallel to $A$. This and $F \in \overline{\operatorname{conv} \operatorname{supp} \nu_{x}}$ prove

$$
\operatorname{supp} \nu_{x} \subseteq F+\operatorname{span} A .
$$

We showed $|C-B|,|D-B| \leq|A|$ and evaluate (6.4), e.g., $W(C)=W^{* *}(C)$. Hence, $|C-B|=|A|=|D-B|$. Consequently, for all $S \in \operatorname{supp} \nu_{x}$ with $|S-B| \leq|A|$, we have $|S-B|=|A|$ and, by (6.5),

$$
S-B=\mathbb{P}(F-B)+t \cdot A_{0} .
$$

From (6.6), we compute $t$ from $|S-B|=|A|$ and find $t= \pm\left(|A|^{2}-|\mathbb{P}(F-B)|^{2}\right)^{1 / 2}$. Compare (4.4) for a similar calculation. This yields $S=S_{+}(F)$ or $S=S_{-}(F)$ and so

$$
\operatorname{supp} \nu_{x} \subseteq\left\{S_{ \pm}(F)\right\} .
$$

Finally, we calculate $\lambda \in[0,1]$ such that $F=\lambda S_{+}(F)+(1-\lambda) S_{-}(F)$ and get $\lambda=\lambda(F)$.

We conclude this section with a summary of few properties of discrete Young measures obtained in a post-processing after the calculation of a discrete solution to the relaxed problem.

Definition 6.2. Let $\nu \in \operatorname{YM}\left(\Omega ; \mathbb{R}^{n}\right)$ be generated by a minimising sequence of $I$ in $\mathcal{A}$ (with $W$ as given in (1.2)) which is weakly convergent to $u$ in $\mathcal{A}$. Define $\xi:=\left(|\nabla u-B|^{2}-|A|^{2}\right)_{+}$and $\Omega_{m}:=\{x \in \Omega: \xi(x)=0\}$.

Let $u_{h}$ be a solution of $\left(R P_{h}\right)$ and set $\nu_{h}:=\mu\left(\nabla u_{h}\right) \in \operatorname{YM}\left(\Omega ; \mathbb{R}^{n}\right)$ and $\xi_{h}:=$ $\left(\left|\nabla u_{h}-B\right|^{2}-|A|^{2}\right)_{+}$. Define

$$
\mathcal{S}(F):= \begin{cases}\left\{S_{ \pm}(F)\right\} & \text { if }|F-B| \leq|A|, \\ F & \text { if }|A|<|F-B|,\end{cases}
$$

and $\operatorname{dist}(X ; Y):=\inf _{(x, y) \in X \times Y}|x-y|$ for two sets $X, Y \subseteq \mathbb{R}^{n}$. Assume $\bigcup_{h} \mathcal{A}_{h}$ is dense in $\mathcal{A}$.

Remark 6.2. (1): $\operatorname{supp} \nu$ is unique, i.e., if $w$ denotes any solution of $(R P)$ we have $\operatorname{supp} \nu_{x}=\mu(\nabla w(x))$ for almost all $x \in \Omega$ (although possibly $u \neq w$ ).

(2): In general, $\nu$ is not unique. But, if $u$ is the unique solution of $(R P)$ (see Theorem 3 for sufficient conditions), then $\nu$ is unique and equals $\mu(\nabla u)$ almost everywhere in $\Omega$.

Proof. Note that $\operatorname{supp} \mu(F)$ depends upon $\mathbb{P}(F-B)$ only and $\mathbb{P}(\nabla u-B)$ is unique by Theorem 3, which shows (1). See Example 1.1 and Proposition 4 for the proof of $(2)$.

Since $\operatorname{supp} \nu \subseteq \mathcal{S}(\nabla u)$ and $\mathcal{S}(\nabla u(x))=\operatorname{supp} \nu_{x}$ for almost all $x \in \Omega$ with $|\nabla u(x)-B| \neq|A|$, the following estimate indicates a strong convergence of the support of $\nu_{h}$ a.e. in $\Omega$ (at least for a subsequence), even if $\nu$ is non-unique. 
Theorem 5. There exists a constant $c_{11}>0$ independent of $h$, such that

$$
\left\|\rho_{h}^{1 / 2} \operatorname{dist}\left(\mathcal{S}\left(\nabla u_{h}\right) ; \mathcal{S}(\nabla u)\right)\right\|_{2} \leq c_{11} \cdot \inf _{v_{h} \in \mathcal{A}_{h}}\left(\alpha\left\|u-v_{h}\right\|_{2}+\left\|\nabla u-\nabla v_{h}\right\|_{4}\right)^{1 / 2},
$$

where the weight $\rho_{h}$ is given by $\rho_{h}(x)=1$ for almost all $x \in \Omega_{m}$ and $\rho_{h}(x)=$ $\min \left\{1, \xi(x)+\xi_{h}(x)\right\}$ for almost all $x \in \Omega \backslash \Omega_{m}$.

Proof. From Theorem 4 we obtain for almost all $x \in \Omega$

$$
\left\|\min \left\{1, \xi_{h}+\xi\right\}^{1 / 2}\left(\nabla u-\nabla u_{h}\right)\right\|_{2} \leq c \cdot \inf _{v_{h} \in \mathcal{A}_{h}}\left(\alpha\left\|u-v_{h}\right\|_{2}+\left\|\nabla u-\nabla v_{h}\right\|_{4}\right) .
$$

Here and throughout this proof, $c$ will denote a generic positive constant independent of $h$.

Since $\mathcal{S}(F)$ is Lipschitz in the sense

$$
\operatorname{dist}(\mathcal{S}(F) ; \mathcal{S}(G)) \leq c|F-G| \quad\left(F, G \in \mathbb{R}^{n}\right),
$$

we conclude from (6.9) that

$$
\begin{aligned}
\| \min & \left\{1, \xi_{h}+\xi\right\}^{1 / 2} \operatorname{dist}\left(\mathcal{S}\left(\nabla u_{h}\right) ; \mathcal{S}(\nabla u)\right) \|_{2} \\
\leq c & \inf _{v_{h} \in \mathcal{A}_{h}}\left(\alpha\left\|u-v_{h}\right\|_{2}+\left\|\nabla u-\nabla v_{h}\right\|_{4}\right) .
\end{aligned}
$$

Hence, it remains to prove that, in addition to (6.10), we have

$$
\left\|\operatorname{dist}\left(\mathcal{S}\left(\nabla u_{h}\right) ; \mathcal{S}(\nabla u)\right)\right\|_{L^{2}\left(\Omega_{h}^{\prime}\right)} \leq c \cdot \inf _{v_{h} \in \mathcal{A}_{h}}\left(\alpha\left\|u-v_{h}\right\|_{2}+\left\|\nabla u-\nabla v_{h}\right\|_{4}\right)^{1 / 2},
$$

where $\Omega_{h}^{\prime}=\left\{x \in \Omega_{m}: \xi_{h}(x)<1\right\}$. To prove (6.11), we consider $x \in \Omega_{h}^{\prime}$ and set $F:=\nabla u(x), F_{h}:=\nabla u_{h}(x)$. We distinguish the two cases $\left|F_{h}-B\right| \leq|A|$ and $|A|<\left|F_{h}-B\right|$. In the case $\left|F_{h}-B\right| \leq|A|$, we have

$$
\begin{aligned}
\operatorname{dist} & \left(\mathcal{S}\left(F_{h}\right) ; \mathcal{S}(F)\right)^{2} \\
& =\left|\mathbb{P}\left(F-F_{h}\right)\right|^{2}+\left|\left[|A|^{2}-|\mathbb{P}(F-B)|^{2}\right]^{1 / 2}-\left[|A|^{2}-\left|\mathbb{P}\left(F_{h}-B\right)\right|^{2}\right]^{1 / 2}\right|^{2} \\
& \leq\left|\mathbb{P}\left(F-F_{h}\right)\right|^{2}+\left.|| \mathbb{P}(F-B)\right|^{2}-\left|\mathbb{P}\left(F_{h}-B\right)\right|^{2} \mid \\
& \leq\left|\mathbb{P}\left(F-F_{h}\right)\right|\left(\left|\mathbb{P}\left(F-F_{h}\right)\right|+|\mathbb{P}(F-B)|+\left|\mathbb{P}\left(F_{h}-B\right)\right|\right) \\
& \leq 4|A|\left|\mathbb{P}\left(F-F_{h}\right)\right|
\end{aligned}
$$

while in the case $\left|F_{h}-B\right|>|A|$, writing $\xi_{h}=\left|F_{h}-B\right|^{2}-|A|^{2}=\left|\mathbb{P}\left(F_{h}-B\right)\right|^{2}+$ $\left|\left(F_{h}-B\right) \cdot A_{0}\right|^{2}-|A|^{2}>0$, we obtain

$$
\begin{aligned}
\operatorname{dist}\left(\mathcal{S}\left(F_{h}\right) ; \mathcal{S}(F)\right)^{2} & =\left|\mathbb{P}\left(F-F_{h}\right)\right|^{2}+\left|\left[|A|^{2}-|\mathbb{P}(F-B)|^{2}\right]^{1 / 2}-\right|\left(F_{h}-B\right)^{T} \cdot A_{0}||^{2} \\
& \leq\left|\mathbb{P}\left(F-F_{h}\right)\right|^{2}+\left.|| A\right|^{2}-|\mathbb{P}(F-B)|^{2}-\left|\left(F_{h}-B\right)^{T} \cdot A_{0}\right|^{2} \mid \\
& =\left|\mathbb{P}\left(F-F_{h}\right)\right|^{2}+\left.|| \mathbb{P}\left(F_{h}-B\right)\right|^{2}-|\mathbb{P}(F-B)|^{2}-\xi_{h} \mid \\
& \leq \xi_{h}+2\left|\mathbb{P}\left(F-F_{h}\right)\right|\left(\left|\mathbb{P}\left(F-F_{h}\right)\right|+|\mathbb{P}(F-B)|\right) \\
& \leq \xi_{h}+2\left|\mathbb{P}\left(F-F_{h}\right)\right|\left(2|\mathbb{P}(F-B)|+\left|\mathbb{P}\left(F_{h}-B\right)\right|\right) \\
& \leq \xi_{h}+2\left|\mathbb{P}\left(F-F_{h}\right)\right|\left(2|A|+\xi_{h}\right) .
\end{aligned}
$$


Noting that $\xi_{h}<1$, we find in both cases that

$$
\operatorname{dist}\left(\mathcal{S}\left(F_{h}\right) ; \mathcal{S}(F)\right)^{4} \leq c\left(\xi_{h}^{2}+\left|\mathbb{P}\left(F-F_{h}\right)\right|^{2}\right) \quad \text { a.e. in } \Omega_{h}^{\prime} .
$$

By Theorem 4 this yields

$$
\left\|\operatorname{dist}\left(\mathcal{S}\left(\nabla u_{h}\right) ; \mathcal{S}(\nabla u)\right)\right\|_{L^{4}\left(\Omega_{h}^{\prime}\right)}^{2} \leq c \cdot \inf _{v_{h} \in \mathcal{A}_{h}}\left(\alpha\left\|u-v_{h}\right\|_{2}+\left\|\nabla u-\nabla v_{h}\right\|_{4}\right),
$$

which implies (6.11).

Remark 6.3. If $u$ is unique, we have weak convergence of the coefficients of the Young measure approximations

$$
\lambda\left(\nabla u_{h}\right) \rightarrow^{*} \lambda(\nabla u) \quad(\text { weak star }) \text { in } L^{\infty}(\Omega) .
$$

\section{A posteriori ERror estimates}

We specify notation for the discretization dealing with conforming finite elements on regular triangulations.

Definition 7.1. Let $\Omega \subset \mathbb{R}^{n}$ have a polyhedral boundary $\Gamma=\Gamma_{0}$. For each $h>0$ let $\mathcal{T}_{h}$ be a regular partition of $\Omega$ into $n$-simplices, called elements, (such that any two simplices are either disjoint or share a complete submanifold of their boundaries). For each element $T \in \mathcal{T}_{h}$, the ratio of the diameter $h_{T}$ of $T$ and the diameter of the largest ball included in $T$ is bounded from above by a universal constant $C_{1}$ independent of $h$. Let $\mathcal{E}_{h}$ be the set of all faces $E$ of an element not belonging to $\Gamma$ and let $h_{E}$ be the diameter of $E \in \mathcal{E}_{h}$.

Let $S_{h}^{k,-1}$ be the set of all (in general discontinuous) functions $v: \Omega \rightarrow \mathbb{R}$ such that $\left.v\right|_{T}$ is a polynomial of (total) degree at most $k$ for all $T \in \mathcal{T}_{h}$. Set $S_{h}^{k, 0}:=S_{h}^{k,-1} \cap C(\bar{\Omega})$ and define

$$
\mathcal{A}_{0 h}:=S_{h}^{k, 0} \cap W_{0}^{1, p}(\Omega)
$$

for some $k \geq 1$. let

Given a minimiser $u_{h}$ of $I^{* *}$ in $\mathcal{A}_{h}:=u_{0}+\mathcal{A}_{0 h}$, let $\sigma_{h}:=\Sigma\left(\nabla u_{h}\right)$. For all $T \in \mathcal{T}_{h}$

$$
\eta_{h}(T):=h_{T}^{p^{\prime}} \cdot \int_{T}\left|g+2 \alpha\left(f-u_{h}\right)+\operatorname{div} \sigma_{h}\right|^{p^{\prime}} d x+\sum_{E \subset \partial T \backslash \Gamma} h_{E} \cdot \int_{E}\left|\left[\sigma_{h} n_{E}\right]\right|^{p^{\prime}} d s .
$$

We assume $p, p^{\prime}$ to be conjugate exponents, i.e. $1 / p+1 / p^{\prime}=1$. The integrand $\left[\sigma_{h} n_{E}\right]$ denotes the jump of the discrete stresses $\sigma_{h} n_{E}$ along a face $E$ of two neighbouring elements, $n_{E}$ is a unit normal vector of a fixed orientation along $E$, and summation in (7.1) is over all such faces of $T$.

The following result implies (1.10) in $\S 1$.

Theorem 6. Assume the hypothesis of Definition 3.1 and (7.1) with $1+s / p \leq t<$

$r$. Then there exists a constant $c_{12}>0$ independent of $h$, such that

$$
\left\|\sigma-\sigma_{h}\right\|_{r / t}^{r}+\alpha\left\|u-u_{h}\right\|_{2}^{2} \leq c_{12} \cdot\left(\sum_{T \in \mathcal{T}_{h}} \eta_{h}(T)\right)^{1-1 / p} .
$$


Proof. Using (3.7) and (3.8) and the Euler-Lagrange equation for the minimiser $u$, one obtains for all $v_{h} \in \mathcal{A}_{h}$,

$$
\begin{aligned}
\frac{1}{c_{9}}\left\|\sigma-\sigma_{h}\right\|_{r / t}^{r}+2 \alpha \int_{\Omega}\left|u-u_{h}\right|^{2} d x \leq & -\int_{\Omega} \sigma_{h} \cdot D\left(u-v_{h}\right) d x \\
& +\int_{\Omega}\left(u-v_{h}\right)\left(g+2 \alpha\left(f-u_{h}\right)\right) d x .
\end{aligned}
$$

Element-wise integration by parts in the first integral proves that the upper bound equals

$$
\sum_{T \in \mathcal{T}_{h}} \int_{T}\left(u-v_{h}\right)\left(g+2 \alpha\left(f-u_{h}\right)+\operatorname{div} \sigma_{h}\right) d x+\sum_{E \in \mathcal{E}_{h}} \int_{E}\left(u-v_{h}\right)\left[\sigma_{h} n\right] d x .
$$

Now we choose an approximation $v_{h} \in \mathcal{A}_{h}$ to $u$ as in [Cle75] and obtain, as mentioned, e.g., in [BS94, Ver94], that

$$
\begin{aligned}
& \left\|u-v_{h}\right\|_{L^{p}(T)} \leq C_{2} h_{T}\|u\|_{W^{1, p}(\mathcal{N}(T))}, \\
& \left\|u-v_{h}\right\|_{L^{p}(E)} \leq C_{3} h_{E}^{1-1 / p}\|u\|_{W^{1, p}(\mathcal{N}(E))}
\end{aligned}
$$

for all $T \in \mathcal{T}_{h}$ and $E \in \mathcal{E}_{h}$ where $\mathcal{N}(T)$ (resp. $\mathcal{N}(E)$ ) is a domain occupied by all neighbouring elements of $T$ including $T$ itself (resp. $E$ ). The constants $C_{2}, C_{3}$ depend only on $C_{1}$ and $k$ but not on $h$. Using this in the upper bound, we get from Hölder's inequality,

$$
\begin{aligned}
\frac{1}{C_{3}} \| \sigma & -\sigma_{h} \|_{r / t}^{r}+2 \alpha \int_{\Omega}\left|u-u_{h}\right|^{2} d x \\
\leq & C_{4}\|u\|_{1, p}\left(\sum_{T \in \mathcal{T}_{h}} h_{T}^{p^{\prime}}\left\|g+\alpha\left(f-u_{h}\right)+\operatorname{div} \sigma_{h}\right\|_{L^{p^{\prime}(T)}}^{p^{\prime}}\right)^{1 / p^{\prime}} \\
& +C_{4}\|u\|_{1, p}\left(\sum_{E \in \mathcal{E}_{h}} h_{E}\left\|\left[\sigma_{h} n\right]\right\|_{L^{p^{\prime}(E)}}^{p^{\prime}}\right)^{1 / p^{\prime}}
\end{aligned}
$$

From this and $\|u\|_{1, p} \leq c_{8}$, cf., the proof of Theorem 2, we conclude (7.2).

Remark 7.1. The above a posteriori estimate is computable with even the constants known in principle. Assuming (3.1)-(3.3) only, we have no hypothesis on the gradients therefore we used the rough estimate $\left\|u-v_{h}\right\|_{1, p} \leq\|u\|_{1, p} \leq c_{8}$. If one had sufficient control on $\left\|u-v_{h}\right\|_{1, p}$, the exponents of $h_{T}$ and $h_{E}$ in (7.1) could be larger and thereby (7.2) could be sharpened.

Remark 7.2. The proofs of Theorems 3,5 and 6 show that, in the case where $W$ is defined by (1.2) and $p=4$,

$$
\begin{array}{r}
\left\|\mathbb{P} \nabla u-\mathbb{P} \nabla u_{h}\right\|_{2}+\left\|\left|\xi+\xi_{h}\right|^{1 / 2} \cdot A_{0}^{T} \cdot\left(\nabla u-\nabla u_{h}\right)\right\|_{2}+\left\|\xi-\xi_{h}\right\|_{2}+\alpha\left\|u-u_{h}\right\|_{2} \\
+\left\|\rho_{h}^{1 / 2} \operatorname{dist}\left(\mathcal{S}\left(\nabla u_{h}\right) ; \mathcal{S}(\nabla u)\right)\right\|_{2}^{2} \leq c_{13} \cdot\left(\sum_{T \in \mathcal{T}_{h}} \eta_{h}(T)\right)^{3 / 8}
\end{array}
$$

\section{Numerical EXPERIMENTS}

A numerical computation of a solution to $\left(R P_{h}\right)$ leads to a minimisation of a discrete problem on a finite dimensional space with a typically large dimension. Hence, we have to take into account sparse structures of matrices that appear in a 
standard descent algorithm. The Truncated Newton Method was shown in [Nas84] efficient for large-scale minimisation problems like $\left(R P_{h}\right)$ :

Algorithm 1 (Algorithm to solve $\left(R P_{h}\right)$ ).

1. Choose $u_{h}^{0}$ and set $j=0$. Until a stopping criterion is fulfilled continue with (2)-(4).

2. Determine a search direction $v_{h}^{j}$ by performing a few steps of a preconditioned conjugate gradient algorithm to solve the linear system

$$
D^{2} I_{h}^{* *}\left(u_{h}^{j}\right) \cdot v_{h}^{j}=-D I_{h}^{* *}\left(u_{h}^{j}\right) .
$$

3. Perform line search by computing (an approximation to) $\alpha^{j} \in \mathbb{R}$ such that

$$
I^{* *}\left(u_{h}^{j}+\alpha^{j} v_{h}^{j}\right)=\min \left\{I^{* *}\left(u_{h}^{j}+\alpha v_{h}^{j}\right): \alpha \in \mathbb{R}\right\} .
$$

4. Update $u_{h}^{j+1}:=u_{h}^{j}+\alpha^{j} v_{h}^{j}, j:=j+1$ and go to (2).

Remark 8.1. As the relaxed problem is strictly convex (for $\alpha>0$ ) the Hessian matrix $D^{2} I_{h}^{* *}\left(u_{h}^{j}\right)$ in (8.1) is positive definite and has a sparse structure because of the underlying $\mathrm{FE}$ approximation. We run a preconditioned conjugate gradient algorithm (PCG) in (2) where the preconditioner is simply the diagonal. Following [Nas84], the PCG iterations are terminated after a number of steps regarding computer effort and energy levels. Hence, in $(2), v_{h}^{j}$ is an approximation between the steepest descent direction (i.e., for one CG step) and Newton-Raphson's corrector (i.e., for infinite CG steps) and is therefore called the truncated Newton method.

In our numerical examples, reported below, 10 to 20 PCG steps were necessary in step (2) while we needed 10 to 30 line searches for any new mesh within a nested iteration (i.e., choose $u_{h}^{0}$ for a finer mesh as the interpolated discrete solution on the coarser mesh). Throughout our numerical experiments the computer effort has grown only linearly with the number of unknowns and so the standard solver based on Algorithm 1 proved to be efficient.

For more details on the method and its implementation (such as approximation and updates of the Hessian which are not stored and computed explicitly) we refer to [Nas84] and references quoted therein.

Our test examples are based on Tartar's example with a broken extremal (see, e.g., [NW92]).

Example 8.1. Let $n=2$ and $\Omega=(0,1)^{2}$. Consider $I$ as given in Definition 1.1 where $W$ is given in $(1.2)$ with $F_{1}=(-1,0)$ and $F_{2}=(1,0)$ and, for

$$
f_{0}(x)=-3 / 128(x-0.5)^{5}-1 / 3(x-1 / 2)^{3},
$$

$f(x, y)=f_{0}(x), g(x, y)=0, \alpha=1$. Then from Tartar's one dimensional example the solution of $(R P)$ is known to be

$$
u(x, y)=f_{1}(x):= \begin{cases}f_{0}(x) & \text { for } 0 \leq x \leq 1 / 2, \\ 1 / 24(x-1 / 2)^{3}+x-1 / 2 & \text { for } 1 / 2 \leq x \leq 1 .\end{cases}
$$

The minimal energy is $E:=\min I^{* *}=1409 / 30000$ and microstructure is present in $\Omega_{m}=(0,1 / 2) \times(0,1)$.

On uniform meshes (here: halved squares) with mesh size $h$, Algorithm 1 leads to approximate solutions $u_{h}$ of $\left(R P_{h}\right)$. In Tables 1 and 2 we displayed for each $h$ the number of degrees of freedoms $N=N_{h}$ and $E_{h}-E, E_{h}:=I^{* *}\left(u_{h}\right)$ is the energy 
TABLE 1. Results on uniform meshes in Example 8.1 (interface known)

\begin{tabular}{|cccccccc|}
\hline$N$ & $h$ & $E_{h}-E$ & $\left\|u-u_{h}\right\|_{L^{2}}$ & $e_{h}:=\left\|u-u_{h}\right\|_{H^{1}}$ & $\eta_{h}$ & $\alpha_{h}\left(e_{h}\right)$ & $\alpha_{h}\left(\eta_{h}\right)$ \\
\hline 25 & .250 & $6.0 \mathrm{e}-05$ & $2.3 \mathrm{e}-03$ & $3.1 \mathrm{e}-02$ & $3.6 \mathrm{e}-01$ & & \\
81 & .125 & $1.5 \mathrm{e}-05$ & $6.0 \mathrm{e}-04$ & $1.6 \mathrm{e}-02$ & $2.6 \mathrm{e}-01$ & 0.97 & 0.44 \\
121 & .100 & $9.2 \mathrm{e}-06$ & $3.8 \mathrm{e}-04$ & $1.3 \mathrm{e}-02$ & $2.4 \mathrm{e}-01$ & 0.98 & 0.46 \\
289 & .062 & $3.6 \mathrm{e}-06$ & $1.5 \mathrm{e}-04$ & $8.0 \mathrm{e}-03$ & $1.9 \mathrm{e}-01$ & 0.98 & 0.47 \\
441 & .050 & $2.3 \mathrm{e}-06$ & $9.6 \mathrm{e}-05$ & $6.4 \mathrm{e}-03$ & $1.7 \mathrm{e}-01$ & 0.96 & 0.48 \\
961 & .033 & $1.0 \mathrm{e}-06$ & $4.3 \mathrm{e}-05$ & $4.4 \mathrm{e}-03$ & $1.4 \mathrm{e}-01$ & 0.93 & 0.48 \\
1089 & .031 & $8.9 \mathrm{e}-07$ & $3.7 \mathrm{e}-05$ & $4.2 \mathrm{e}-03$ & $1.4 \mathrm{e}-01$ & 0.90 & 0.49 \\
2601 & .020 & $3.6 \mathrm{e}-07$ & $1.5 \mathrm{e}-05$ & $2.9 \mathrm{e}-03$ & $1.1 \mathrm{e}-01$ & 0.84 & 0.49 \\
\hline
\end{tabular}

TABLE 2. Results on uniform meshes in Example 8.1 (interface unknown)

\begin{tabular}{|cccccccc|}
\hline$N$ & $h$ & $E_{h}-E$ & $\left\|u-u_{h}\right\|_{L^{2}}$ & $e_{h}:=\left\|u-u_{h}\right\|_{H^{1}}$ & $\eta_{h}$ & $\alpha_{h}\left(e_{h}\right)$ & $\alpha_{h}\left(\eta_{h}\right)$ \\
\hline 324 & .059 & $1.2 \mathrm{e}-05$ & $2.2 \mathrm{e}-03$ & $1.2 \mathrm{e}-01$ & $1.9 \mathrm{e}-01$ & & \\
784 & .037 & $3.4 \mathrm{e}-06$ & $1.1 \mathrm{e}-03$ & $9.6 \mathrm{e}-02$ & $1.5 \mathrm{e}-01$ & 0.50 & 0.48 \\
1444 & .027 & $1.5 \mathrm{e}-06$ & $6.7 \mathrm{e}-04$ & $8.2 \mathrm{e}-02$ & $1.3 \mathrm{e}-01$ & 0.50 & 0.49 \\
2304 & .021 & $8.1 \mathrm{e}-07$ & $4.7 \mathrm{e}-04$ & $7.3 \mathrm{e}-02$ & $1.1 \mathrm{e}-01$ & 0.50 & 0.49 \\
3364 & .018 & $5.0 \mathrm{e}-07$ & $3.5 \mathrm{e}-04$ & $6.6 \mathrm{e}-02$ & $1.0 \mathrm{e}-01$ & 0.50 & 0.49 \\
\hline
\end{tabular}

of (the computed approximation to) $u_{h} ; E$ is the minimal energy. The numerical results prove that the energy $E_{h}$ is decreasing and seemingly convergent to $E$ as $h \rightarrow 0$.

Since the solution $u$ is known in Example 8.1, we computed the errors and reported the decreasing error norms $\left\|u-u_{h}\right\|_{2}$ and $e_{h}:=\left\|u-u_{h}\right\|_{1,2}$ in Tables 1 and 2 as well.

Note that the mesh sizes in Table 1 are such that the boundary of $\Omega_{m}$ coincides with element boundaries. Although the solution $u$ is only piecewise smooth, $u$ is smooth on each element whence

$$
\inf _{v_{h} \in \mathcal{A}_{h}}\left\|u-v_{h}\right\|_{1,4}=O(h) .
$$

Therefore, the a priori estimates in Theorem 4 predict linear convergence of $u_{h}$ to $u$ in a certain weighted energy-like norm. To compare this with the numerical results we compute the experimental convergence rate

$$
\alpha_{h}\left(e_{h}\right):=-\log \left(e_{h} / e_{H}\right) / \log \left(N_{h}^{1 / 2} / N_{H}^{1 / 2}\right)
$$

where $N_{h}, e_{h}$ and $N_{H}, e_{H}$ are number of degrees of freedom and errors on two successive meshes. The quantity $N_{h}^{1 / 2}$ corresponds to $h$ for uniform meshes and is used for comparisons with results on non-uniform meshes. Then Table 1 suggests that we have even linear convergence of the errors in the energy norm.

On the other hand the meshes in Table 2 are such that the boundary of $\Omega_{m}$ does not coincide with element boundaries. Hence, (8.2) cannot be expected and, indeed, Table 2 indicates a convergence rate $1 / 2$ only. 
For an a posteriori error control we computed

$$
\eta_{h}(T):=h_{T}^{4 / 3}\left\|g+2 \alpha\left(f-u_{h}\right)+\operatorname{div} \sigma_{h}\right\|_{L^{4 / 3}(T)}^{4 / 3}+\frac{1}{2} \sum_{E \subset \partial T \cap \Omega} h_{E}\left\|\left[\sigma_{h} \cdot n_{E}\right]\right\|_{L^{4 / 3}(E)}^{4 / 3}
$$

for each element $T$ in $\mathcal{T}_{h}$ and then

$$
\eta_{h}:=\left(\sum_{h \in \mathcal{T}_{h}} \eta_{h}(T)\right)^{3 / 8} .
$$

According to (1.10) and 7.3 we have, e.g.,

$$
\left\|\sigma-\sigma_{h}\right\|_{4 / 3}+\left\|u-u_{h}\right\|_{2}+\left\|\mathbb{P} \nabla u-\mathbb{P} \nabla u_{h}\right\|_{2}+\left\|\xi-\xi_{h}\right\|_{2} \leq c \cdot \eta_{h} .
$$

From Tables 1 and 2, we observe that $\eta_{h}$ is decreasing which indicates convergence owing to (8.6). The experimental convergence rates $\alpha_{h}\left(\eta_{h}\right)$ of $\eta_{h}$ (defined in a similar way to $(8.3)$ ) are also shown and indicate $1 / 2$ in both cases. This coincides with the convergence rate of the energy norms in Table 2 and suggests that, the worst-case estimate (8.6) cannot, in general, be sharpened.

So far we considered uniform meshes. Because of a possibly different behaviour of relaxed solutions in $\Omega_{m}$ and in $\Omega \backslash \overline{\Omega_{m}}$ we may be interested in an adaptive automatic mesh refinement to improve the approximation property of the discrete spaces: in Example 8.1 a mesh refinement towards the line $\{1 / 2\} \times(0,1)$ (where $u$ is non-smooth). Since the coefficients $\eta_{h}(T)$ are local (in the sense that they are computable for each element) they may serve to steer the mesh refinement. According to the literature (e.g., [Ver94]), we applied the following adaptive scheme.

Algorithm 2 (Algorithm for adaptive mesh refinement).

1. Start with a coarse initial mesh $\mathcal{T}_{h_{0}}$, set $k=0$.

2. Solve the discrete problem $u_{h_{k}}$ on the mesh $\mathcal{T}_{h_{k}}$.

3. Compute $\eta_{h_{k}}(T)$ for each $T$ in $\mathcal{T}_{h_{k}}$ as in (8.4).

4. Compute the upper error bound (4.1) and decide to stop (then terminate computation) or to refine (then go to (5)).

5. Refine (i.e., halve the largest edge of) $T \in \mathcal{T}_{h_{k}}$ provided

$$
\eta_{h_{k}}(T) \geq \frac{1}{2} \cdot \max _{T^{\prime} \in \mathcal{T}_{h_{k}}} \eta_{h_{k}}\left(T^{\prime}\right)
$$

6. Refine further triangles to avoid hanging nodes and thereby create a new mesh $\mathcal{T}_{h_{k+1}}$. Update $k$ to $k+1$ and go to $(2)$.

In Example 8.1 we run Algorithm 2 starting with different uniform meshes whose element sides do not coincide with $\{1 / 2\} \times(0,1)$ (situation as in Table 2 ). The generated meshes are refined almost uniformly in $\Omega \backslash \Omega_{m}$ and near to the interface. To compress the output we draw the results for $e_{h}$ and $\eta_{h}$ in Fig. 2 where $\log \left(N_{h}^{1 / 2}\right)$ is plotted against $-\log \left(e_{h}\right)$. According to the $\log$-scaling, an algebraic convergence of order $\alpha$ is displayed by a slope $\alpha$.

From Fig. 2 one observes that the convergence behaviour of the meshes is considerably improved by Algorithm 2, where c (resp. d) refers to numerical results obtained with a regular (irregular) initial mesh (non-aligned with $\Omega_{m}$ in both cases).

In the second example we were interested in a more general situation, where the exact solution as well as $\Omega_{m}$ are not known. 


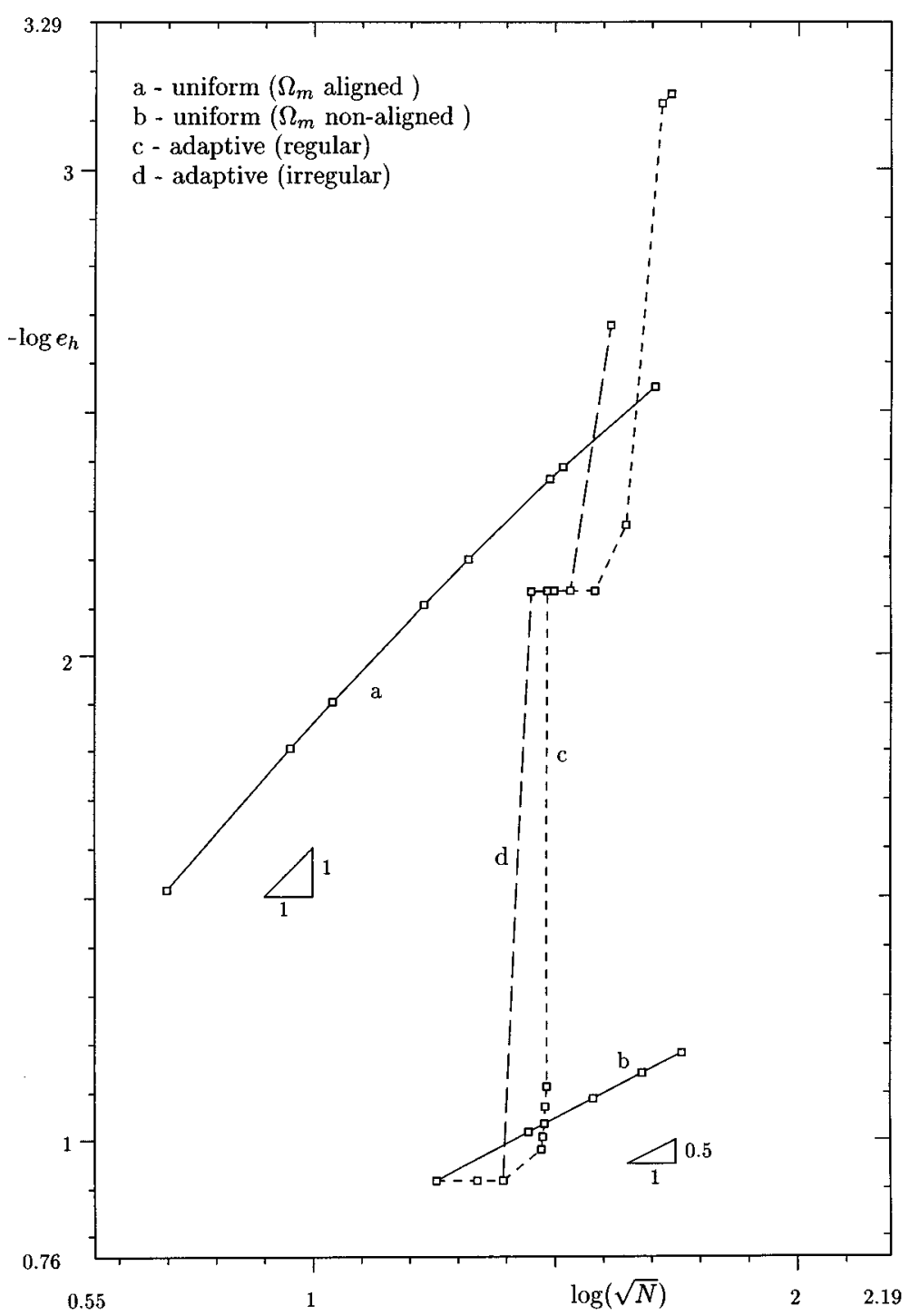

FiguRE 2. Errors $e_{h}:=\left\|u-u_{h}\right\|_{1,2}$ in Example 8.1 for uniform and adapted meshes

Example 8.2. Rotate the wells of $W$ by $\phi=4.7746483$ and so let $F_{1}:=-F_{2}:=$ $-(\cos \phi, \sin \phi)$. Define $f(x, y):=f_{0}(x \cos \phi+y \sin \phi)$ and the boundary condition $u_{0}(x, y)=f_{1}(x \cos \phi+y \sin \phi)$ on $\partial \Omega$ and adopt the remaining notation from Example 8.1.

Since a priori information is lacking, we rely on a posteriori error estimates and computed $\eta_{h}$ as explained above for various uniform and adaptive meshes displayed in Fig. 3. As seen there, the upper bound $\eta_{h}$ is decreasing and so indicates convergence with an experimental convergence rate $\alpha=1 / 2$. Again, the meshes automatically generated by Algorithm 2 appear superior to the uniform meshes. 


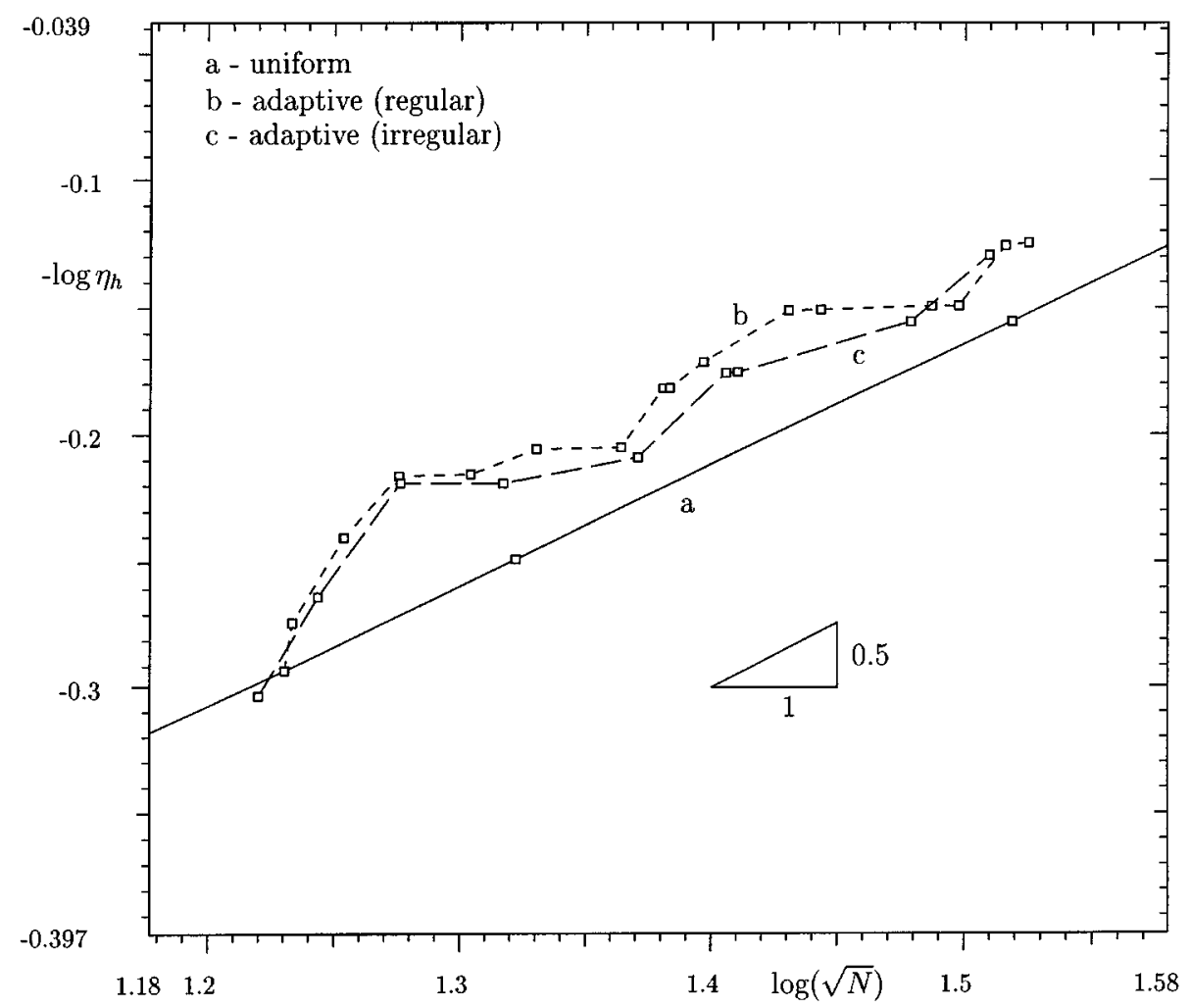

FiguRE 3. A posteriori estimates $\eta_{h}$ in Example 8.2 for uniform and adapted meshes

From this a posteriori information we infer that the numerical results obtained in our computations are reasonable: The deformation field $u_{h}$, the stress field $\sigma_{h}$ and the volume fractions $\lambda_{h}:=\lambda\left(\nabla u_{h}\right)$ (as defined in (6.1)) are plotted in Figs. 4, 5 and 6 for the finest mesh (also indicated in Fig. 4) with $N=1122$ and 2147 elements. This mesh was generated by Algorithm 2 for $k=6$ starting with a regular coarse mesh which is a submesh of the finest mesh displayed in Fig. 7, where the approximate microstructure region is shown as well. One observes mesh refinements towards the boundary of $\Omega_{h m} \cap \Omega$.

For comparison we also ran Algorithm 2 with a different irregular mesh and obtained the mesh and the microstructure region $\Omega_{m h}$ in Fig. 8, where the approximation to $\Gamma_{m}:=\partial \Omega_{m} \cap \Omega$ is emphasised. The approximation of $\Gamma_{m}$ is very similar in Fig. 7 and 8 and indicates that $\Gamma_{m}$ may be a curve (which is an open question). In both cases we have a high refinement near $\Gamma_{m}$ suggesting some non-smoothness of the solution $u$ which is not obvious from the displacement plot in Fig. 4 .

To compare our results with a direct minimisation of Problem $(P)$ we calculated an approximation to a discrete minimiser (at least some approximation to a solution of the discrete Euler-Lagrange equations) as shown in Fig. 9 on a fine uniform mesh with $h=1 / 20, E_{1 / 20}=1.38432, N=441$; also shown is an enlargement of a portion of the solution on a finer mesh with $h=1 / 80, E_{1 / 80}=1.31266, N=6561$. 


\section{Deformation}

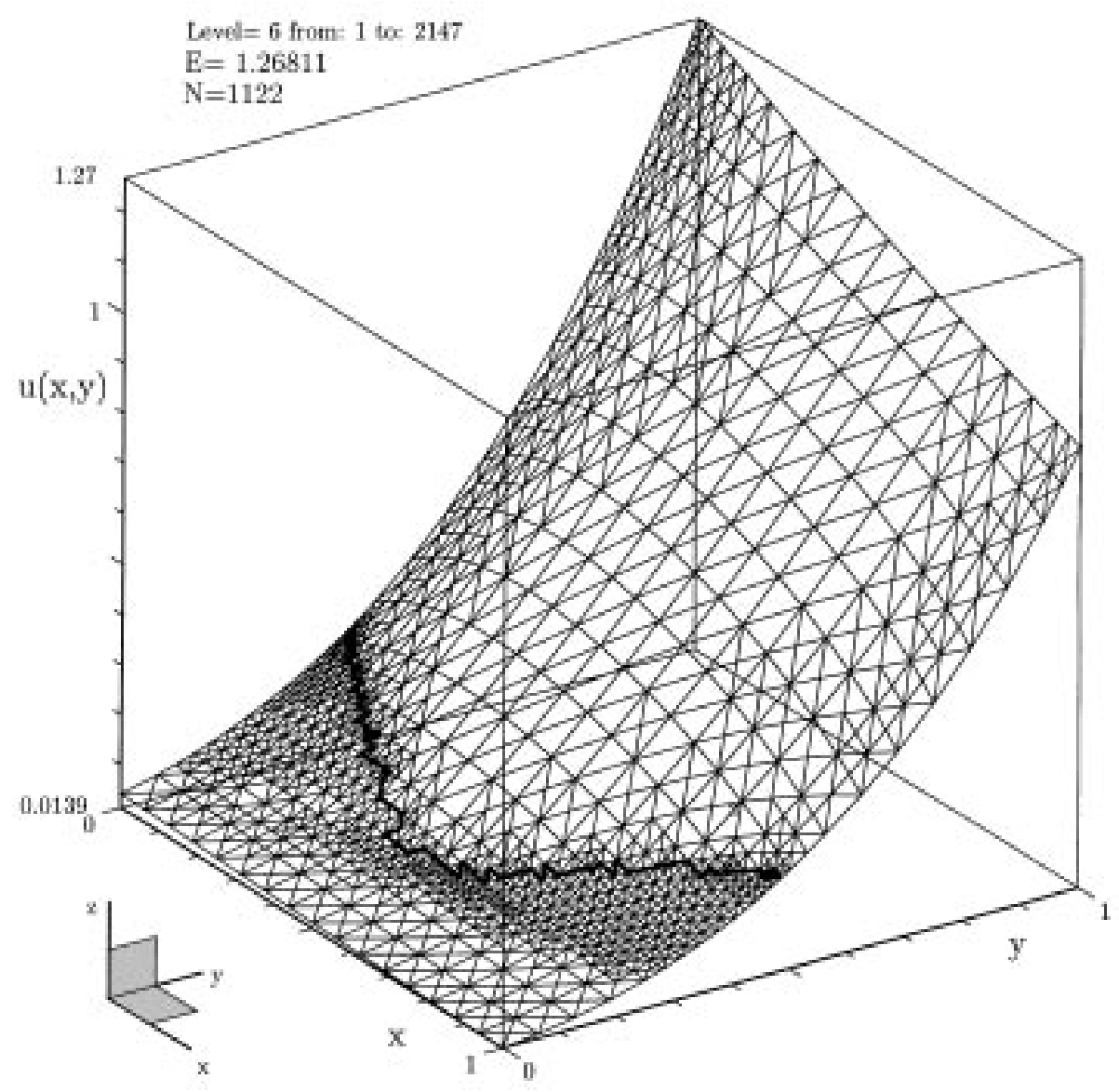

Figure 4. Deformation $u_{h}$ in Example 8.2

The macroscopic displacement field seems to coincide with the one in Fig. 4 while we see oscillations depicting $\Omega_{m}$. However, the microstructure region predicted by Fig. 9 is not sharp. As the energy for $\left(P_{h}\right)$ with $N=6561$ is much bigger than $E_{h}=1.26811$ obtained for $\left(R P_{h}\right)$ with $N=1122$ we cannot exclude the possibility that there is a better discrete minimiser of $\left(P_{h}\right)$, it is uncertain if the numerical result is reliable.

To summarise the comparison, the relaxed problem is easier and cheaper to solve, the numerical results appear more stable, detailed and reasonable. Moreover the method is applicable to any geometry and with irregularly adapted meshes. 


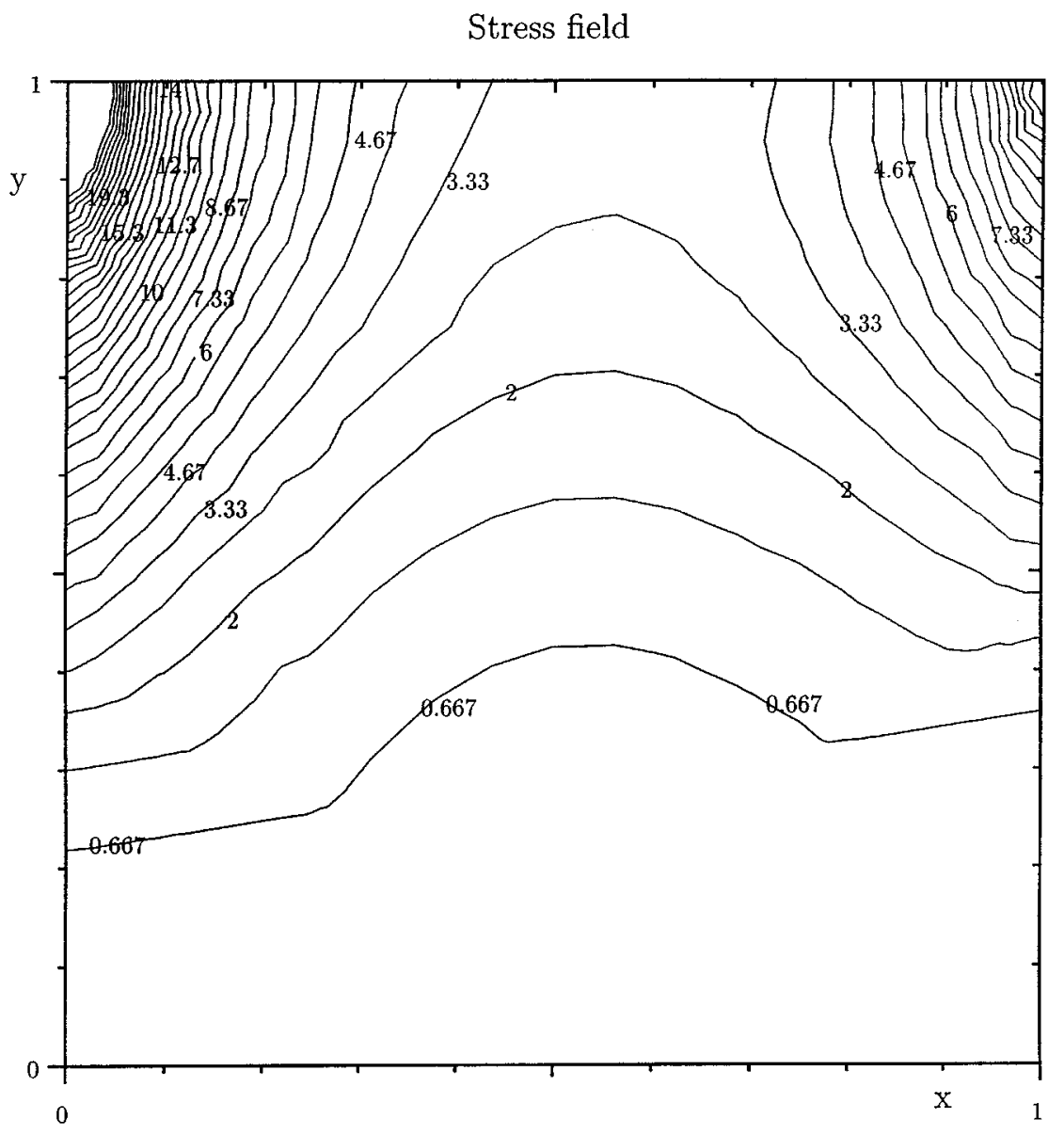

Figure 5. Stress field $\left|\sigma_{h}\right|$ in Example 8.2 
Volume fractions

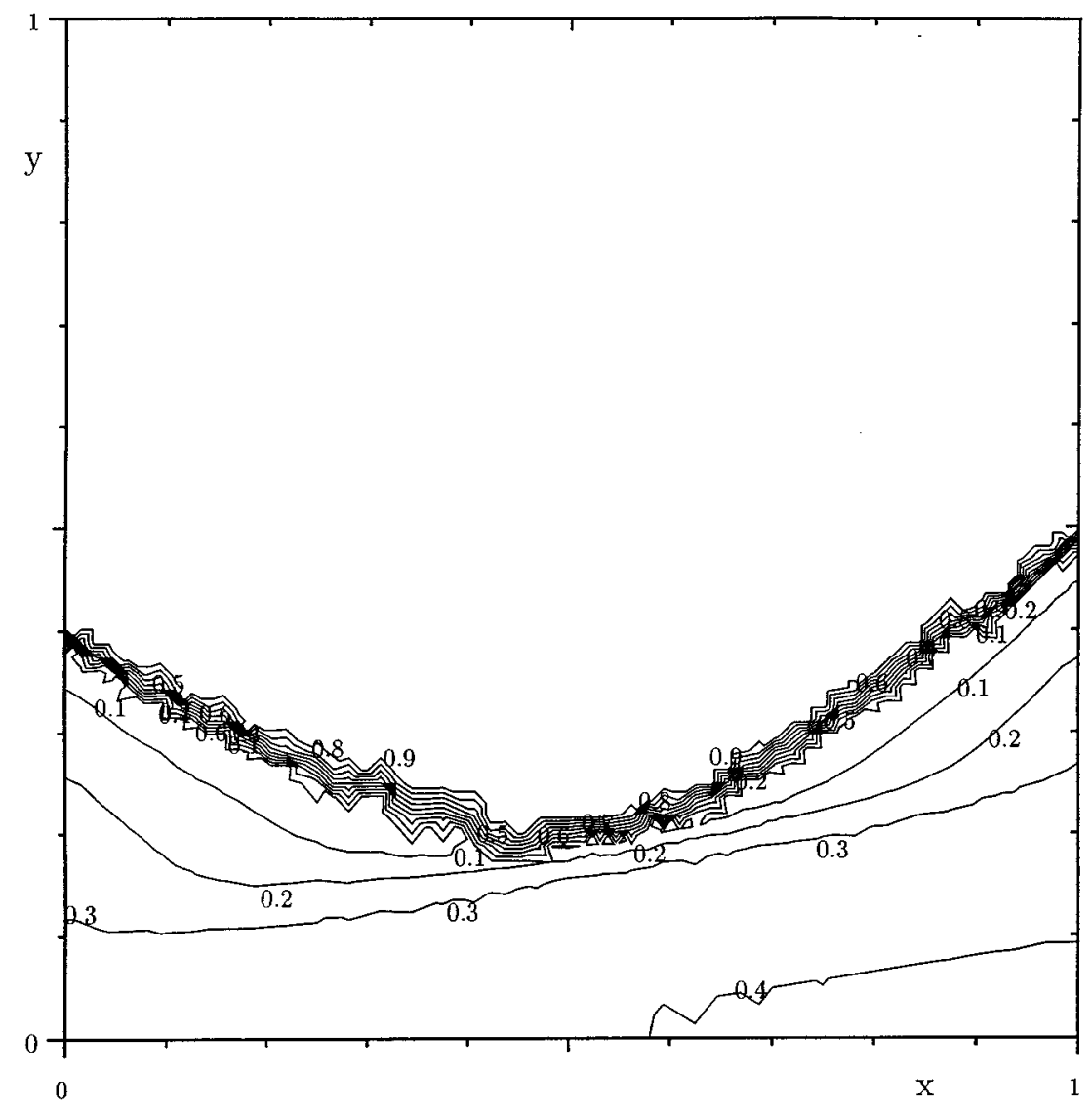

FiguRE 6. Volume fraction $\lambda_{h}$ in Example 8.2 


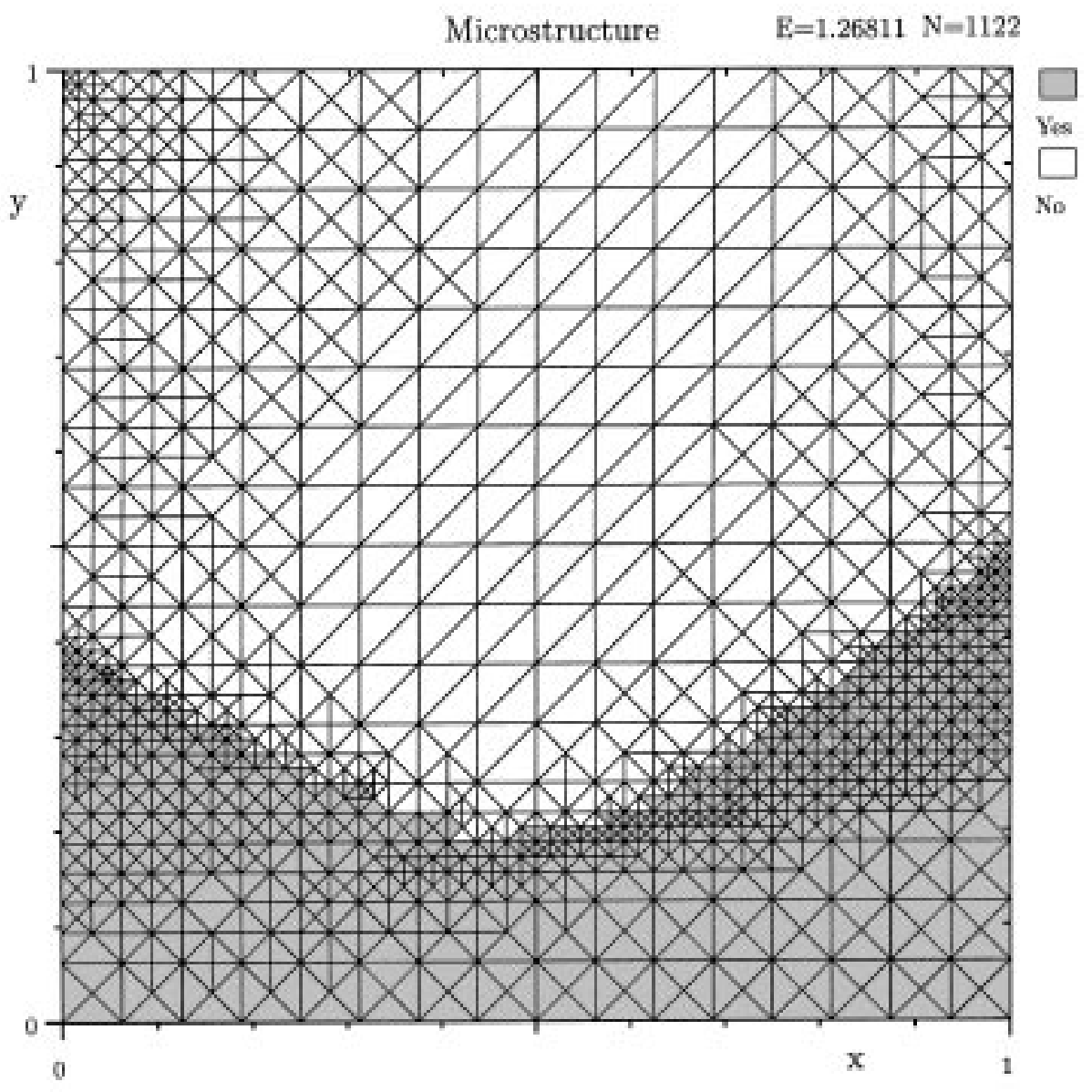

Figure 7. Plot of $\Omega_{m h}$ in Example 8.2 


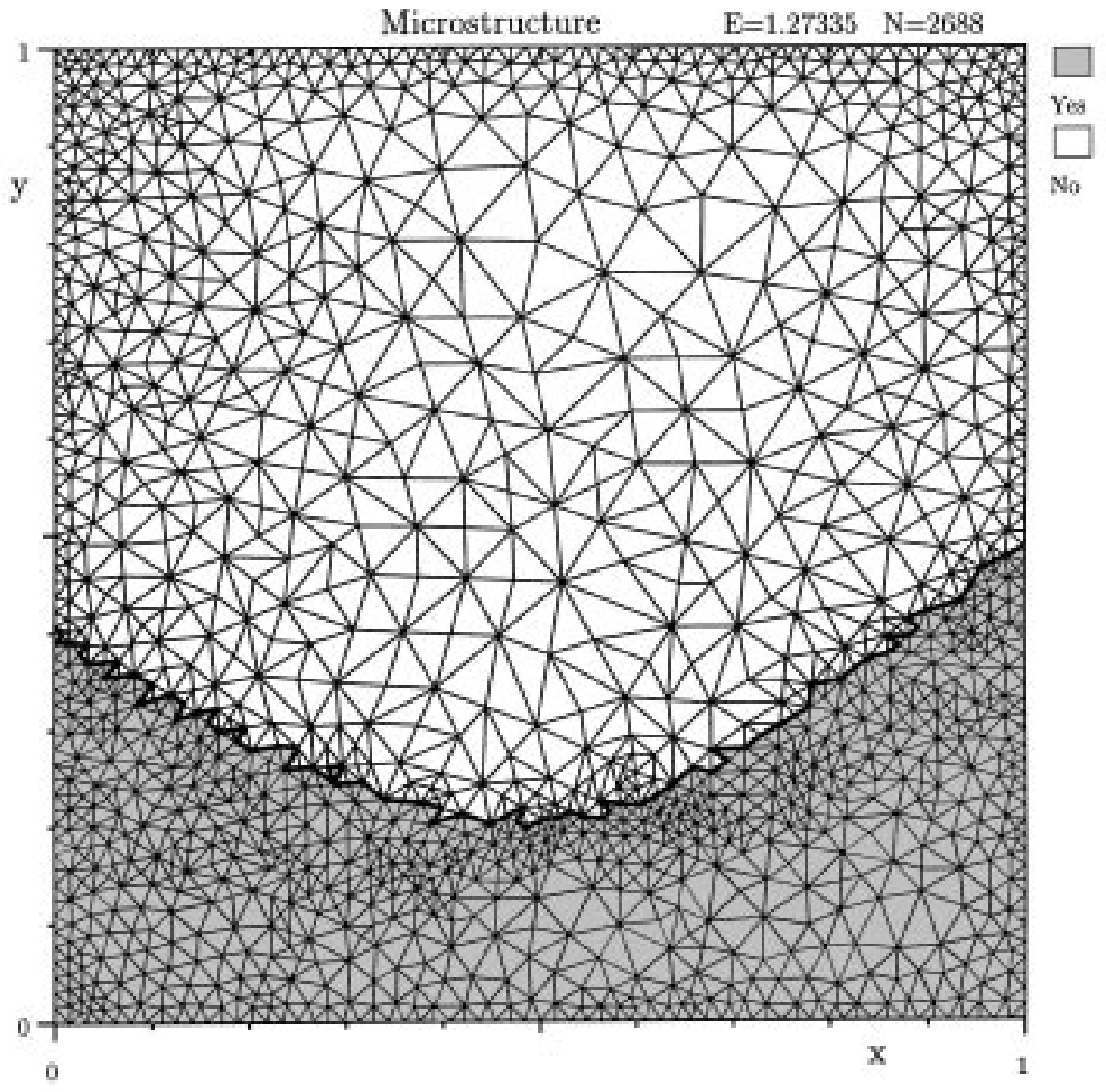

FiguRE 8. Microstructure on adaptively refined irregular mesh in Example 8.2 

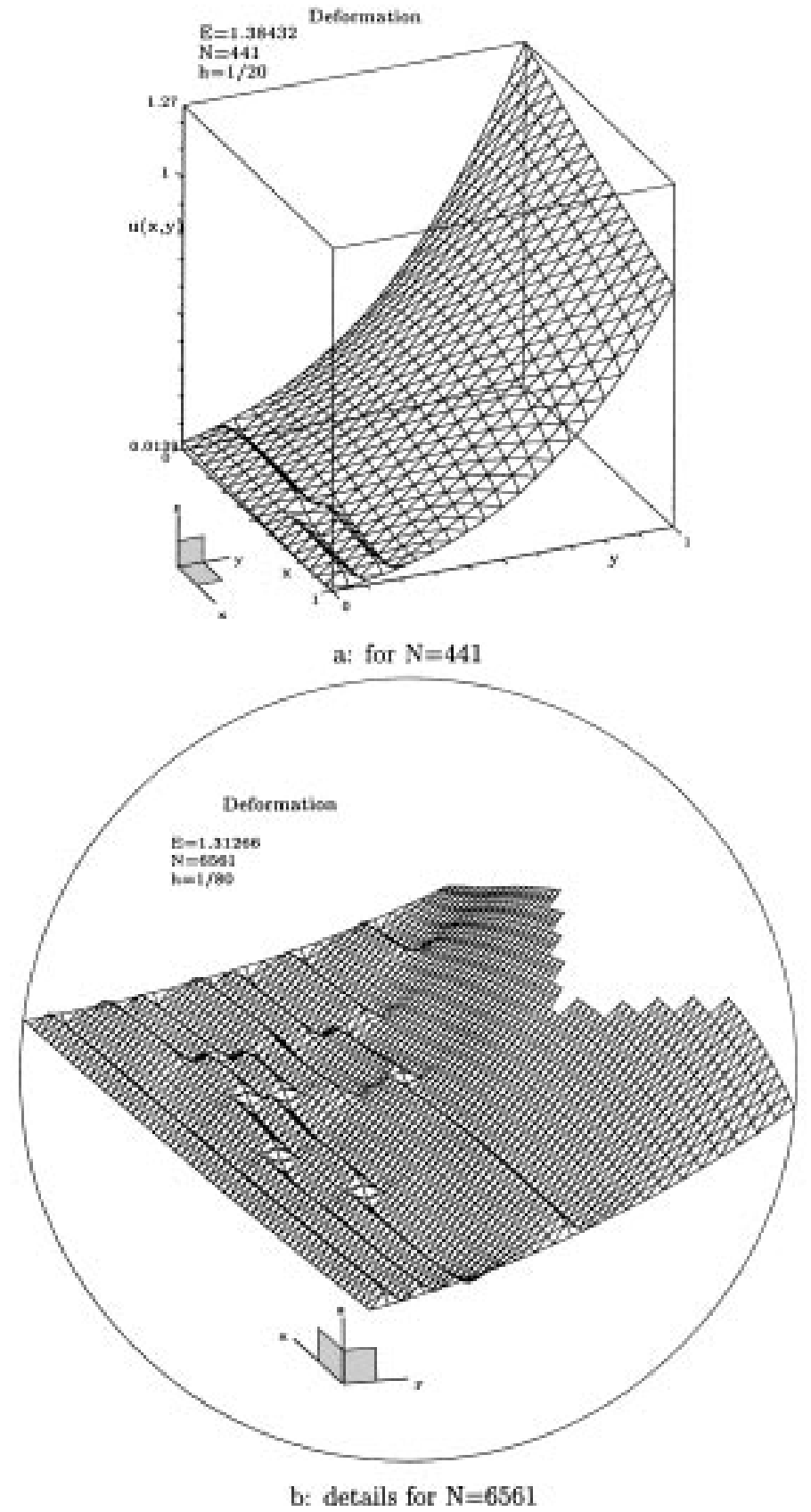

FiguRE 9. Deformation $u_{h}$ in Example 8.2 for the non-relaxed problem $\left(P_{h}\right)$ 


\section{ACKNOWLEDGEMENT}

It is our pleasure to thank John M. Ball for stimulating discussions.

\section{REFERENCES}

[Bal89] J.M. Ball, A version of the fundamental theorem for Young measures, In M Rascle, D. Serre, and M. Slemrod, editors, Partial differential equations and continuum models of phase transitions, LNP 344, pages 207-215, 1989. MR 91c:49021

[BC94] B. Brighi and M. Chipot, Approximated convex envelope of a function, SIAM J. Numer. Anal., 31, 1994. MR 94m:49049

[BJ87] J. M. Ball and R. D. James, Fine phase mixtures as minimisers of energy, Arch. Rational Mech. Anal., 100:13-52, 1987. MR 89c:80005

[BJ92] J. M. Ball and R. D. James, Proposed experimental tests of the theory of fine microstructure and the two-well problem, Phil. Trans. R. Soc. Lond. A., 338:389-450, 1992.

[BP90] P. Bauman and D. Phillips, A nonconvex variational problem related to change of phase, Appl. Math. Optimization, 21:113-138, 1990. MR 90i:49004

[BS94] S. C. Brenner and L. R. Scott, The mathematical theory of finite element methods, volume 15 of Texts in Applied Mathematics, Springer Verlag, New York, 1994. MR 95f: 65001

[CC92] M. Chipot and C. Collins, Numerical approximations in variational problems with potential wells, SIAM J. Numer. Anal., 29:1002-1019, 1992. MR 93j:65171

[Chi91] M. Chipot, Numerical analysis of oscillations in nonconvex problems, Numer. Math., 59:747-767, 1991. MR 92h:65164

[CL91] C. Collins and M. Luskin, Optimal order error estimates for the finite element approximation of a solution of a nonconvex variational problem, Math. Comp., 57:621-637, 1991. MR 92a:65276

[Cle75] P. Clement, Approximation by finite element functions using local regularization, RAIRO Sér. Rouge Anal. Numér., R-2:77-84, 1975. MR 53:4569

[Dac89] B. Dacorogna, Direct methods in the calculus of variations, Springer, Berlin, 1989. MR 90e: 49001

[DK91] P. Pedregal D. Kinderlehrer, Characterisations of Young measures generated by gradients, Arch. Rat. Mech. Anal., 115:329-365, 1991. MR 92k:49089

[EG92] L. C. Evans and R. F. Gariepy, Measure theory and fine properties of functions, CRC Press, 1992. MR 92f:28001

[Fre90] D. French, On the convergence of finite element approximations of a relaxed variational problem, SIAM J. Num. Anal., 27:419-436, 1990. MR 91f:65167

[Fri94] G. Friesecke, A necessary and sufficient condition for non-attainment and formation of microstructure almost everywhere in scalar variational problems, Proc. R. Soc. Edin, 124A:437-471, 1994. MR 96g:49001

[GKR86] J. Goodman, R. V. Kohn, and L. Reyna, Numerical study of a relaxed variational problem from optimal design, Comput. Methods Appl. Mech. Engrg., 57:107-127, 1986. MR 88a:73054

[GT81] M. E. Gurtin and R. Temam, On the anti-plane shear problem in elasticity, J. Elasticity, 11:197-206, 1981. MR 82d:73030

[KP91] D. Kinderlehrer and P. Pedregal, Weak convergence of integrands and the Young measure representation, SIAM J Math. Anal., 23:1-19, 1991. MR 92m:49076

[Nas84] S. Nash, Newton-type minimisation via the Lanczos method, SIAM J. Num. Anal., 21:770-787, 1984. MR 86h:65092

[NW92] R. A. Nicolaides and N. J. Walkington, Computation of microstructure utilizing Young measure representations, In C.A. Rogers and R.A. Rogers, editors, Recent Advances in Adaptive and Sensory Materials and their Applications, pages 131-141, Lancaster, 1992. Technomic Publishing Co.

[NW95] R. A. Nicolaides and N. J. Walkington, Strong convergence of numerical solutions to degenerate variational problems, Math. Comp., 64:117-127, 1995. MR 95m:65183

[Ped92] P. Pedregal, Jensen's inequality in calculus of variations, Differential Integral Equations, 7:57-72, 1994. MR 94i: 49032 
[Rou] T. Roubíček, Relaxation in optimization theory and variational calculus, DeGruyter, Berlin 1997.

[Ver94] R. Verfürth, A posteriori error estimates for nonlinear problems. Finite element discretization of elliptic equations, Math. Comp., 62:445-475, 1994. MR 94j:65136

Mathematisches Seminar II der Christian-Albrechts-Universität ZU Kiel， D-24098 Kiel, Germany

E-mail address: cc@numerik.uni-kiel.de

Mathematical Institute, Oxford University, 24-29 St. Giles, Oxford OX1 3LB, UNITED Kingdom 